KEK-TH-922

hep-th/0310228

\title{
D-branes of Covariant AdS Superstrings
}

\author{
Makoto Sakaguchi $^{a}$ and Kentaroh Yoshida ${ }^{b}$ \\ Theory Division, High Energy Accelerator Research Organization (KEK), \\ Tsukuba, Ibaraki 305-0801, Japan. \\ ${ }^{a}$ Makoto.Sakaguchi@kek.jp $\quad{ }^{b}$ kyoshida@post.kek.jp
}

\begin{abstract}
We consider D-branes of open superstrings in the $\mathrm{AdS}_{5} \times S^{5}$ background. The possible configurations of D-branes preserving half of supersymmetries are classified by analyzing the $\kappa$-invariance of an open superstring in a covariant manner. We also revisit the classification of D-branes in the pp-wave background. It is shown that Penrose limits of the possible D-branes in the $\mathrm{AdS}_{5} \times S^{5}$ give all of the D-branes in the pp-wave. In addition, a $1 / 4$ supersymmetric D-string, which is related to the D-string preserving 8 dynamical supersymmetries in the ppwave, is presented. We also discuss the relation between our result and the AdS branes in a brane probe analysis.
\end{abstract}

Keywords: D-branes, AdS string, AdS branes, Wess-Zumino term, $\kappa$-symmetry, Penrose limit, pp-wave 


\section{Introduction}

The discovery of D-branes [1] has played an important role for revealing non-perturbative aspects of superstring theories and M-theory [2]. Recent interest in studies of D-branes is the classification of possible configurations in non-trivial backgrounds. Among other non-trivial backgrounds, the maximally supersymmetric type IIB pp-wave background [3] has attracted great interests because the Green-Schwarz superstring on this background was shown to be exactly solvable $[4,5]$. The configurations of D-branes on pp-wave backgrounds were examined in many works [6-22]. In particular, covariant analyses of D-branes of an open string in ppwaves were given in [14,19] by applying a method developed by Lambert and West [23].

It is also possible to apply this method for analyzing Dirichlet branes of an open supermembrane. In the eleven-dimensional flat spacetime, Dirichlet $p$-branes were shown to be allowed for the values $p=1,5,9[24,25]$. The $p=5$ case corresponds to M5-brane, while the $p=9$ case describes the end-of-world 9-brane in the Horava-Witten theory [26]. Studies of open supermembrane theories are expected to promote our understanding of features of M-branes.

Dirichlet branes of an open supermembrane on the maximally supersymmetric pp-wave background (Kowalski-Glikman solution)* were classified in [30,32]. In the work [32], we provided a covariant classification of the possible D-brane configurations by combining methods in Refs. [24,25] and Ref. [23]. We found 1/2 supersymmetric M5-brane and 9-brane configurations sitting at the origin of the pp-wave. These configurations are not $1 / 2$ supersymmetric outside the origin while 1-brane configurations are 1/2 supersymmetric at and outside the origin. Also, 1/2 supersymmetric 9-brane configurations obtained in Ref. [32] are expected to be available for a study of a heterotic matrix model [33]. If we consider a double dimensional reduction of open supermembrane on the pp-wave background to a type IIA string theory [13] through a compactification of one of transverse dimensions, then M5- and 9-branes in eleven dimensions wrapping an $S^{1}$-circle become possible configurations of D4-branes and D8-branes in the resulting type IIA pp-wave background $[13,16,19,20]$. This correspondence strongly supports the weak-strong duality between M-theory and type IIA string theory even in pp-wave backgrounds, and thus we can say that a consistency of superstring theories on a pp-wave has been checked.

In our previous work [34], Dirichlet branes of an open supermembrane in the $\mathrm{AdS}_{4 / 7} \times S^{7 / 4}$

${ }^{*}$ Supermembrane on the pp-wave background is related to the pp-wave matrix model [27] through the matrix regularization [28]. This relation in the pp-wave case is discussed in [29-31]. 
backgrounds were examined, and possible configurations were classified. It was shown that under the Penrose limit [35] possible Dirichlet branes are mapped one-to-one to those on the pp-wave background, as expected from the fact that the Kowalski-Glikman solution [36] can be obtained from the $\mathrm{AdS}_{4 / 7} \times S^{7 / 4}$ backgrounds [37]. The allowed Dirichlet branes are also related to AdS branes in the $\mathrm{AdS}_{4 / 7} \times S^{7 / 4}$ backgrounds.

Motivated by our previous works [32,34], we will consider D-branes of an AdS string by analyzing the covariant Wess-Zumino term. First, we classify D-brane configurations of an open superstring in the $\mathrm{AdS}_{5} \times S^{5}$ background. The possible D-brane configurations can be compared to the AdS brane configurations. Secondly, we extend the covariant classification of D-branes in the pp-wave [14] by including the cases that the null directions $(+,-)$ are Dirichlet ones. Thirdly, we examine Penrose limits of the possible D-branes in the $\operatorname{AdS}_{5} \times S^{5}$ background, and directly show that all of D-branes in the pp-wave are realized in these limits. In addition, we present an example of $1 / 4$ supersymmetric D-string configurations in the $\mathrm{AdS}_{5} \times S^{5}$ background, which preserves $1 / 4$ supersymmetries even outside the origin. This configuration reduces to the $1 / 4$ supersymmetric D-string in the pp-wave background found in the work [14] through the Penrose limit. From the viewpoint of embedded branes in the $\operatorname{AdS}_{5} \times S^{5}$ background, this D-string may possibly correspond to $R \times S^{1}$.

The organization of our paper is as follows: In Section 2, we introduce the action of an open superstring on the $\mathrm{AdS}_{5} \times S^{5}$ background, and the covariant Wess-Zumino term is presented. In section 3 , we classify possible $1 / 2$ supersymmetric D-brane configurations of the AdS string by investigating the vanishing conditions of the $\kappa$-variation surface terms of the covariant WessZumino term. In addition, we find a 1/4 supersymmetric D-string configuration. This D-string preserves a quarter of supersymmetries both at and outside the origin. In section 4, we classify D-brane configurations preserving half of supersymmetries in the pp-wave background which include the cases that the null directions $(+,-)$ satisfy the Dirichlet conditions. In section 5 we consider the Penrose limit of the result obtained in section 3. All of the resulting configurations after the Penrose limit are shown to be included in the list presented in section 4 . We also discuss the relation between our result and $\mathrm{AdS}$ branes in the $\mathrm{AdS}_{5} \times S^{5}$ background. Section 5 is devoted to a conclusion and discussions. In appendix A, our notation and convention are summarized. 


\section{Covariant Wess-Zumino Term for the AdS String}

In this section, we will introduce the Green-Schwarz action of a superstring on the $\operatorname{AdS}_{5} \times S^{5}$ background (called AdS string below), and explain some characteristics relevant to our later considerations. In this paper, we do not utilize any specific gauges but discuss in a covariant fashion, because the covariant expression of the Wess-Zumino term is available for the covariant classification of D-branes of the AdS string.

First of all, the action of AdS string we consider is written as

$$
S=\int d^{2} \sigma\left[\mathcal{L}_{\mathrm{NG}}+\mathcal{L}_{\mathrm{WZ}}\right], \quad \mathcal{L}_{\mathrm{NG}}=-\sqrt{-g(X, \theta)}
$$

The Nambu-Goto part of this Lagrangian is represented in terms of the induced metric $g_{i j}$, which is given by (For notation and convention, see Appendix A)

$$
g_{i j}=E_{i}^{M} E_{j}^{N} G_{M N}=E_{i}^{A} E_{j}^{B} \eta_{A B}, \quad g=\operatorname{det} g_{i j}, \quad E_{i}^{A}=\partial_{i} Z^{\hat{M}} E_{\hat{M}}^{A},
$$

where $Z^{\hat{M}}=\left(X^{M}, \theta^{\bar{\alpha}}\right)$ and $E_{\hat{M}}^{A}$ are supervielbeins of the $\mathrm{AdS}_{5} \times S^{5}$ background. For D-strings, $g$ is replaced with $\operatorname{det}\left(g_{i j}+\mathcal{F}_{i j}\right)$ where $\mathcal{F}$ is defined by $\mathcal{F}=d A-B$ with the Born-Infeld $U(1)$ gauge field $A$ and the pull-back of the NS-NS two-form $B$.

The Wess-Zumino term is described as ${ }^{\dagger}$

$$
\mathcal{L}_{\mathrm{WZ}}=-2 i \int_{0}^{1} d t \widehat{E}^{A} \bar{\theta} \Gamma_{A} \sigma \widehat{E},
$$

where $\widehat{E}^{A} \equiv E^{A}(t \theta)$ and $\widehat{E}^{\alpha} \equiv E^{\alpha}(t \theta)$. When we consider a fundamental string (F-string), the matrix $\sigma$ is given by $\sigma_{3}$. If we consider a D-string, then $\sigma$ is represented by $\sigma_{1}$. Since we would like to discuss boundary surfaces for both of fundamental string and D-string, we do not explicitly fix $\sigma$ in our consideration. It is known that $\kappa$-invariance of the action is equivalent to supergravity equations of motion $[41,42]$. In the case of an open string, the $\kappa$-variation leads to surface terms. One can show that the surface terms originating from $S_{\mathrm{NG}}$ vanish, because they include $\delta_{\kappa} Z^{\hat{M}} E_{\hat{M}}^{A}=\delta_{\kappa} E^{A}=0$, by using the definition of the $\kappa$-variation $\delta_{\kappa} E^{A}=0$. In addition, the $\kappa$-variation of $\mathcal{F}$ does not lead to surface terms because $\delta_{\kappa} \mathcal{F}$ does not include a derivative of the $\kappa$-variation of a field. Thus non-vanishing surface terms originate from $S_{\mathrm{WZ}}$ only.

\footnotetext{
${ }^{\dagger}$ Alternative superstring actions have been proposed in [38] for superstrings in the AdS background and in $[39,40]$ for those in the pp-wave background.
} 
In this section, we consider an open string in the $\mathrm{AdS}_{5} \times S^{5}$ background. The concrete expressions of the supervielbein and the spin connection are given in Appendix B where the coset construction is briefly reviewed for this background. We examine the $\kappa$-variation surface terms up to and including the fourth order of $\theta$ in this paper ${ }^{\ddagger}$. After short calculation, the Wess-Zumino term can be rewritten as

$$
S_{\mathrm{WZ}}=S_{\mathrm{WZ}}^{0}+S_{\mathrm{WZ}}^{\mathrm{spin}}+S_{\mathrm{WZ}}^{\mathcal{M}}
$$

where each part in the above decomposition is defined as, respectively,

$$
\begin{aligned}
S_{\mathrm{WZ}}^{0} \equiv & -2 i \int d^{2} \sigma \epsilon^{i j}\left[-\frac{1}{2} \bar{\theta} \Gamma_{A} \sigma\left(\partial_{i} \theta+\frac{\lambda}{2} \widehat{\Gamma}_{B} i \sigma_{2} \theta \partial_{i} X^{M} e_{M}^{B}\right) \partial_{j} X^{N} e_{N}^{A}\right. \\
& \left.+\frac{i}{4} \bar{\theta} \Gamma^{A}\left(\partial_{i} \theta+\frac{\lambda}{2} \widehat{\Gamma}_{B} i \sigma_{2} \theta \partial_{i} X^{M} e_{M}^{B}\right) \cdot \bar{\theta} \Gamma_{A} \sigma\left(\partial_{j} \theta+\frac{\lambda}{2} \widehat{\Gamma}_{C} i \sigma_{2} \theta \partial_{j} X^{N} e_{N}^{C}\right)\right] \\
S_{\mathrm{WZ}}^{\mathrm{spin}} \equiv & -2 i \int d^{2} \sigma \epsilon^{i j}\left[-\frac{1}{8} \bar{\theta} \Gamma_{A} \sigma \Gamma_{B C} \theta \omega_{M}^{B C} \partial_{i} X^{M} \partial_{j} X^{N} e_{N}^{A}\right. \\
& +\frac{i}{16} \bar{\theta} \Gamma^{A} \Gamma_{B C} \theta \omega_{M}^{B C} \partial_{i} X^{M} \cdot \bar{\theta} \Gamma_{A} \sigma\left(\partial_{j} \theta+\frac{\lambda}{2} \widehat{\Gamma}_{D} i \sigma_{2} \theta \partial_{j} X^{N} e_{N}^{D}\right) \\
& +\frac{i}{16} \bar{\theta} \Gamma^{A}\left(\partial_{i} \theta+\frac{\lambda}{2} \widehat{\Gamma}_{B} i \sigma_{2} \theta \partial_{i} X^{M} e_{M}^{B}\right) \cdot \bar{\theta} \Gamma_{A} \sigma \Gamma_{C D} \theta \omega_{N}^{C D} \partial_{j} X^{N} \\
& \left.+\frac{i}{64} \bar{\theta} \Gamma^{A} \Gamma_{B C} \theta \omega_{M}^{B C} \partial_{i} X^{M} \cdot \bar{\theta} \Gamma_{A} \sigma \Gamma_{D E} \theta \omega_{N}^{D E} \partial_{j} X^{N}\right] \\
S_{\mathrm{WZ}}^{\mathcal{M}} \equiv & -2 i \int d^{2} \sigma \epsilon^{i j}\left[-\frac{1}{24} \bar{\theta} \Gamma_{A} \sigma \mathcal{M}^{2} D_{i} \theta \partial_{j} X^{M} e_{M}^{A}\right] .
\end{aligned}
$$

This is the covariant Wess-Zumino term, which will be used for a covariant classification of Dbranes in the $\operatorname{AdS}_{5} \times S^{5}$ background in the next section. The sketch of our strategy is as follows: We will consider surface terms of $\kappa$-variation of the above Wess-Zumino term, and investigate the boundary conditions under which all of the surface terms vanish. Then these boundary conditions lead to a classification of possible D-branes. It is worth mentioning that the surface terms do not cancel out each other. Hence, without loss of generality, we can examine the $\kappa$-variation of surface terms separately in each part.

\footnotetext{
${ }^{\ddagger}$ It is expected that the fourth order analysis is sufficient as we will briefly comment later.
} 


\section{Classification of Supersymmetric D-branes of AdS String}

Here let us investigate the boundary conditions under which the $\kappa$-invariance holds. Then we will classify D-brane configurations preserving half of supersymmetries.

\subsection{Boundary Conditions of Covariant String}

Before going to the concrete analysis, we need to introduce the boundary conditions. The openstring world-sheet $\Sigma$ has one-dimensional boundary $\partial \Sigma$. We can impose the Neumann condition and Dirichlet condition on this boundary $\partial \Sigma$. These boundary conditions are represented by

$$
\begin{array}{ll}
\partial_{\mathbf{n}} X^{\bar{A}} \equiv \partial_{\mathbf{n}} X^{M} e_{M}^{\bar{A}}=0 & \text { (Neumann condition) }, \\
\partial_{\mathbf{t}} X^{\underline{A}} \equiv \partial_{\mathbf{t}} X^{M} e^{\frac{A}{M}}=0 & \text { (Dirichlet condition) },
\end{array}
$$

where we have used the overline as $\bar{A}_{i}(i=0, \ldots, p)$ for the indices of Neumann coordinates and the underline as $\underline{A}_{j}(j=p+1, \ldots, 9)$ for the indices of Dirichlet coordinates. The operator $\partial_{\mathbf{t}}$ is a tangential derivative $\partial / \partial \tau$ and $\partial_{\mathbf{n}}$ is a normal derivative $\partial / \partial \sigma$ on the boundary $\partial \Sigma$, respectively. We impose the boundary condition on the fermionic variable $\theta$ :

$$
P^{ \pm} \theta=\theta, \quad P^{ \pm}=\frac{1}{2}(\mathbb{I}+M) .
$$

The gluing matrix $M$ is a product of the $S O(1,9)$ gamma matrices tensored with Pauli matrices, and its concrete form will be fixed so that the $\kappa$-variation surface terms vanish.

\subsection{D-branes of AdS Strings at the Origin}

In this subsection, we examine the $\kappa$-variation of $S_{\mathrm{WZ}}^{0}$. The $\kappa$-variation is defined by $\delta_{\kappa} E^{A}=0$, i.e.

$$
\delta_{\kappa} X^{M}=-i \bar{\theta} \Gamma^{M} \delta_{\kappa} \theta+O\left(\theta^{4}\right) .
$$

One finds that the $\kappa$-variation of $S_{\mathrm{WZ}}^{0}$ is

$$
\begin{aligned}
\delta_{\kappa} S_{\mathrm{WZ}}^{0}= & \int_{\partial \Sigma} d \xi\left[-i \bar{\theta} \Gamma_{\bar{A}} \sigma \delta_{\kappa} \theta \cdot \partial_{\mathbf{t}} X^{M} e_{M}^{\bar{A}}+\frac{1}{2}\left(\bar{\theta} \Gamma^{\underline{A}} \delta_{\kappa} \theta \cdot \bar{\theta} \Gamma_{\underline{A}} \sigma+\bar{\theta} \Gamma_{\underline{A}} \sigma \delta_{\kappa} \theta \cdot \bar{\theta} \Gamma^{A}\right) \partial_{\mathbf{t}} \theta\right. \\
& -\frac{\lambda}{4}\left\{2\left(\bar{\theta} \Gamma_{\bar{A}} \widehat{\Gamma}_{\bar{B}} \sigma i \sigma_{2} \theta \cdot \bar{\theta} \Gamma^{\bar{B}} \delta_{\kappa} \theta-\bar{\theta} \Gamma_{\bar{B}} \widehat{\Gamma}_{\bar{A}} \sigma i \sigma_{2} \theta \cdot \bar{\theta} \Gamma^{\bar{B}} \delta_{\kappa} \theta\right)\right. \\
& \left.\left.+\left(\bar{\theta} \Gamma^{\bar{B}} \delta_{\kappa} \theta \cdot \bar{\theta} \Gamma_{\bar{B}} \widehat{\Gamma}_{\bar{A}} \sigma i \sigma_{2} \theta-\bar{\theta} \Gamma^{\underline{B}} \widehat{\Gamma}_{\bar{A}} i \sigma_{2} \theta \cdot \bar{\theta} \Gamma_{\underline{B}} \sigma \delta_{\kappa} \theta\right)\right\} \partial_{\mathbf{t}} X^{M} e_{M}^{\bar{A}}\right]+O\left(\theta^{6}\right) .
\end{aligned}
$$


First, let us consider the first line. In order for this line to vanish, the following two conditions have to be satisfied:

$$
\bar{\theta} \Gamma_{\bar{A}} \sigma \delta_{\kappa} \theta=0 \quad \text { and } \quad \bar{\theta} \Gamma^{A} \delta_{\kappa} \theta=0
$$

We find that the condition (3.6) is satisfied if we define the projection operator (3.3) with gluing matrices

$$
\begin{aligned}
& M=\left\{\begin{array}{c}
m \otimes i \sigma_{2}, \quad d=2(\bmod 4) \quad p=-1,3,7 \\
m \otimes \rho, \quad d=4(\bmod 4) \quad p=1,5,9
\end{array}\right. \\
& m=s \Gamma^{\underline{A}_{1}} \ldots \Gamma^{A_{d}}, \quad s=\left\{\begin{array}{ll}
1 & \text { for } X^{0}: \text { Neumann } \\
i & \text { for } X^{0}: \text { Dirichlet }
\end{array}, \quad \rho=\left\{\begin{array}{ll}
\sigma_{1} & \text { when } \sigma=\sigma_{3} \\
\sigma_{3} & \text { when } \sigma=\sigma_{1}
\end{array},\right.\right.
\end{aligned}
$$

where the inclusion of the factor $i$ for the case that the time direction $X^{0}$ is a Dirichlet one is equivalent to performing the Wick rotation and considering in the Euclidean formulation. This procedure does not affect the analysis below. These conditions imply that, for the $\sigma=\sigma_{3}$ case, D $p$-branes exist for the values $p=-1,1,3,5,7,9$, while if we consider the $\sigma=\sigma_{1}$ case, then $\mathrm{D} p$-branes with $p=1$ and 5 are replaced with F1- and NS5-branes respectively ${ }^{\S}$. Hence, we obtain the well-known conditions for branes in type IIB string theory in flat spacetime. It is not clear whether the S-duality holds for superstrings in the $\mathrm{AdS}_{5} \times S^{5}$ and pp-wave or not, but it should exist for branes even in these backgrounds. Therefore, we will discuss the D-string case in parallel as well as the fundamental string in the following consideration.

Next, we consider the second and third lines. Because these lines are proportional to the parameter $\lambda$ characterizing the AdS geometry, these give conditions intrinsic to the branes in the $\mathrm{AdS}_{5} \times S^{5}$ background. In order to make these lines vanish, we have to impose two additional conditions:

$$
\bar{\theta} \Gamma_{\bar{A}} \widehat{\Gamma}_{\bar{B}} \sigma i \sigma_{2} \theta=\bar{\theta} \Gamma^{\underline{B}} \widehat{\Gamma}_{\bar{A}} i \sigma_{2} \theta=0
$$

Let us classify the configurations satisfying the boundary conditions (3.8) .

For the $d=2(\bmod 4)$ case, the conditions $(3.8)$ are satisfied for the following case:

- The number of Dirichlet directions in the $\operatorname{AdS}_{5}$ coordinates $\left(X^{0}, \cdots, X^{4}\right)$ is even, and the same condition is also satisfied for the $S^{5}$ coordinates $\left(X^{5}, \cdots, X^{9}\right)$.

\footnotetext{
$\S_{\text {For }} p=9$, the boundary condition is $\theta_{1}=\theta_{2}$ when $\sigma=\sigma_{3}$, while $\theta_{2}=0$ when $\sigma=\sigma_{1}$.
} 
On the other hand, for the $d=4(\bmod 4)$ case, the following conditions should be imposed:

- The number of Dirichlet directions in the $\operatorname{AdS}_{5}$ coordinates $\left(X^{0}, \cdots, X^{4}\right)$ is odd, and the same condition is also satisfied for the $S^{5}$ coordinates $\left(X^{5}, \cdots, X^{9}\right)$.

These conditions restrict the directions to which a brane world-volume can extend, while (3.6) restricts the dimension of the world-volume. The D-brane configurations satisfying the conditions (3.6) and (3.8) are summarized in Tab. 1.

\begin{tabular}{|c|c|c|c|c|c|}
\hline D-instanton & D-string & D3-brane & D5-brane & D7-brane & D9-brane \\
\hline \hline$(0,0)$ & $(0,2),(2,0)$ & $(1,3),(3,1)$ & $(2,4),(4,2)$ & $(3,5),(5,3)$ & absent \\
\hline
\end{tabular}

Table 1: The possible 1/2 supersymmetric D-branes in $\mathrm{AdS}_{5} \times S^{5}$ sitting at the origin.

We found that D-strings are allowed to exist as 1/2 supersymmetric objects, which correspond to $1 / 2$ supersymmetric D-strings in the pp-wave background as will be seen in section 5. On the other hand, it is known that there exist $1 / 4$ supersymmetric D-strings preserving 8 dynamical supersymmetries in the pp-wave [14]. In subsection 3.4, we will present a $1 / 4$ supersymmetric D-string which is the AdS origin of the $1 / 4$ supersymmetric D-string found there. We found that D9-branes are not allowed to exist. As will be seen in section 4, D9-branes are not also allowed in the pp-wave [6,7]. Since our discussion contains the cases that the time direction satisfies the Dirichlet condition, we were able to find the D-instanton configuration.

Notably, our result agrees with the classification of branes embedded in the $A d S_{5} \times S^{5}$ background (called AdS branes) [8], and thus all of D-branes obtained here possibly correspond to the AdS branes. Such a correspondence emerges in the case of Dirichlet branes of an open supermembrane in the $\operatorname{AdS}_{4 / 7} \times S^{7 / 4}$ backgrounds, where the classification of Dirichlet branes [34] agrees with that of AdS branes obtained in [43].

We have discussed the $\kappa$-variation of $S_{\mathrm{WZ}}^{0}$ until now. We have to take account of the $\kappa$ variations of $S_{\mathrm{WZ}}^{\text {spin }}$ and $S_{\mathrm{WZ}}^{\mathcal{M}}$. As we will see later, these parts have no effect on the above classification at the origin. Therefore we can say that the classification of the possible D-brane configurations at the origin has been completely accomplished. For D-branes sitting outside the origin, the $\kappa$-variation of $S_{\mathrm{WZ}}^{\mathrm{spin}}$ leads to additional conditions. On the other hand, $S_{\mathrm{WZ}}^{\mathcal{M}}$ does not affect the results in both at and outside the origin. The discussion concerning these points will be given in the following subsection. 


\subsection{D-branes of AdS Strings outside the Origin}

Here we will consider the contribution of $S_{\mathrm{WZ}}^{\mathrm{spin}}$ part. The $\kappa$-variation of $S_{\mathrm{WZ}}^{\mathrm{spin}}$ is written as

$$
\begin{aligned}
\delta_{\kappa} S_{\mathrm{WZ}}^{\mathrm{spin}}=\frac{i}{2} \int_{\partial \Sigma} & {\left[-\frac{i}{4}\left\{\bar{\theta} \Gamma^{\bar{A}} \delta_{\kappa} \theta \cdot \bar{\theta} \Gamma_{\bar{A}} \sigma \Gamma_{B C} \theta-\bar{\theta} \Gamma^{\underline{A}} \Gamma_{B C} \theta \cdot \bar{\theta} \Gamma_{\underline{A}} \sigma \delta_{\kappa} \theta\right\} \omega \frac{B C}{\bar{D} C} d X^{\bar{D}}\right.} \\
& \left.-\frac{i}{2} \bar{\theta} \Gamma_{\bar{A}} \sigma \Gamma_{B C} \theta \cdot \bar{\theta} \Gamma^{\bar{D}} \delta_{\kappa} \theta \cdot \omega \frac{B C}{D} d X^{\bar{A}}\right] .
\end{aligned}
$$

In order to make the above surface terms vanish, we need the following conditions:

$$
\bar{\theta} \Gamma_{\bar{A}} \Gamma_{B C} \sigma \theta \cdot \omega \frac{B C}{D}=\bar{\theta} \Gamma^{A} \Gamma_{B C} \theta \cdot \omega \frac{B C}{D}=0 .
$$

These conditions are trivially satisfied at the origin (i.e., $X^{\underline{A}}=0$ ), while no configurations, except for D-instantons, satisfy these conditions outside the origin.

We found that $1 / 2$ supersymmetric $\mathrm{D} p$-branes $(p>0)$ are not allowed except for branes sitting at the origin. This is because the homogeneity is not manifest in the coordinate system we took here. The same situation emerges in the case of branes in the pp-wave. There, a flat brane sitting at (outside) the origin of the Brinkmann coordinate system is mapped to a planer (less supersymmetric curved) brane in the Rosen coordinate system [9]. So, it is expected that a $1 / 2$ supersymmetric brane sitting outside the origin has a non-trivial shape in general. On the other hand, we found that D-instantons are allowed to sit even outside the origin as in flat spacetime. This may be related to the fact that a D-instanton world-volume is a point in any coordinate system. The same situation arises in the pp-wave case as we will see later.

We can expect less supersymmetric D-branes such as $1 / 4$ supersymmetric D-branes to exist even outside the origin as discussed in Refs. [8,9]. We will present this type of D-string later.

The remaining task is to examine whether the $S_{\mathrm{WZ}}^{\mathcal{M}}$ part affects the classification at and outside the origin or not. The $\kappa$-variation of $S_{\mathrm{WZ}}^{\mathcal{M}}$ is given by

$$
\delta_{\kappa} S_{\mathrm{WZ}}^{\mathcal{M}}=\frac{\lambda}{12} \int_{\partial \Sigma}\left[\bar{\theta} \Gamma_{\bar{A}} \sigma \widehat{\Gamma}_{\bar{B}} i \sigma_{2} \theta \cdot \bar{\theta} \Gamma^{\bar{B}} \delta_{\kappa} \theta-\frac{1}{2} \bar{\theta} \Gamma_{\bar{A}} \sigma \Gamma_{B C} \theta \cdot \bar{\theta} \widehat{\Gamma}^{B C} i \sigma_{2} \delta_{\kappa} \theta\right] d X^{\bar{A}} .
$$

The above surface terms vanish under the following conditions:

$$
\bar{\theta} \Gamma_{\bar{A}} \widehat{\Gamma}_{\bar{B}} \sigma i \sigma_{2} \theta=\bar{\theta} \widehat{\Gamma}^{\bar{B}} \underline{\underline{C}} i \sigma_{2} \delta_{\kappa} \theta=0,
$$

which are nothing but those obtained in the analysis of the $\kappa$-variation of the $S_{\mathrm{WZ}}^{0}$ part. Thus no new conditions arise, and hence the $S_{\mathrm{WZ}}^{0}$ part has no effect on our classification. 
As a final remark in this section, we comment on higher order terms. Until now we have studied the D-brane configurations up to and including fourth order in $\theta$. We expect that the higher order terms do not lead to any new additional conditions. In other words, the conditions we have obtained in this paper are expected to be sufficient and all surface terms will vanish under these conditions even in the full theory including all orders of $\theta$. In fact, some arguments for the higher order terms were given in Ref. [14].

\section{$3.41 / 4$ Supersymmetric D-string}

We can obtain 1/4 supersymmetric D-branes in addition to those preserving half of supersymmetries. Here we present a configuration of $1 / 4$ supersymmetric D-string as an example.

Since we would like to study a D1-brane configuration, let us consider the following gluing matrix:

$$
M=m \otimes \sigma_{1}, \quad m \equiv \Gamma^{1 \cdots 8} .
$$

in order to pass the conditions in flat space (3.6). This gluing matrix does not automatically satisfy the conditions (3.8) specific to the AdS geometry, and so we need to impose additional conditions:

$$
\widehat{\Gamma}_{0} \sigma_{2} \theta=\theta, \quad \widehat{\Gamma}_{9} \sigma_{2} \theta=\theta
$$

Here it should be noted that the above two conditions are not independent because of the condition $\theta=M \theta$. We can easily rewrite these conditions as

$$
\Gamma^{1 \cdots 8} \theta_{1}=\theta_{1}, \quad \Gamma^{1 \cdots 8} \theta_{2}=\theta_{2}
$$

By the use of the relations $\Gamma^{+-1 \cdots 8}=\Gamma^{11}$ and $\Gamma^{11} \theta_{1,2}=\theta_{1,2}$, we obtain

$$
\Gamma^{-} \Gamma^{+} \theta_{1}=0, \quad \Gamma^{-} \Gamma^{+} \theta_{2}=0 .
$$

When the spinors $\theta_{1}$ and $\theta_{2}$ are decomposed as $\theta_{1,2}=\frac{1}{2} \Gamma^{-} \Gamma^{+} \theta_{1,2}+\frac{1}{2} \Gamma^{+} \Gamma^{-} \theta_{1,2} \equiv \theta_{1,2}^{(+)}+\theta_{1,2}^{(-)}$, the conditions (3.16) implies $\theta_{1,2}^{(+)}=0$ and $\theta_{1,2}=\theta_{1,2}^{(-)}$. Finally, we obtain the expressions of additional conditions:

$$
\Gamma^{+} \theta_{1}=\Gamma^{+} \theta_{2}=0
$$

This condition reduces the number of the remaining supersymmetries, and the D-string configuration we considered here preserves quarter of supersymmetries. 
Moreover, we can see that the $S_{\mathrm{WZ}}^{\mathcal{M}}$ part has no effect on this D-string configuration since the conditions derived from this part is identical with (3.8). We can also check that the $\kappa^{-}$ variation of $S_{\mathrm{WZ}}^{\text {spin }}$ vanishes. The two conditions (3.10) ensure that the surface terms originating from the $\kappa$-variation of $S_{\mathrm{WZ}}^{\mathrm{spin}}$ vanish. By the use of the relation $\left(\Gamma^{1 \cdots 8} \otimes \mathbb{I}_{2}\right) \theta=\theta$, we can see that the first one in (3.10) is satisfied, while the second one is not satisfied. However, the term $\bar{\theta} \Gamma_{\underline{A}} \sigma \delta_{\kappa} \theta$ that couples to the second condition vanishes under the condition $\left(\Gamma^{1 \cdots 8} \otimes \mathbb{I}_{2}\right) \theta=\theta$, and

hence the surface terms from $S_{\mathrm{WZ}}^{\mathrm{spin}}$ vanish. Thus, we have found a $1 / 4$ supersymmetric D-string configuration in the $\mathrm{AdS}_{5} \times S^{5}$ background. In particular, the supersymmetries preserved by this configuration are not changed even if it is slid from the origin. If we consider the Penrose limit of this D-string, then we can recover the D-string configuration originally found in the work [14], which is $1 / 4$ supersymmetric even outside the origin. The D-string in the $\operatorname{AdS}_{5} \times S^{5}$ might correspond to $R \times S^{1}$ mentioned in the works on the brane probe analysis given in [8]. It is an interesting future work to delve into other branes preserving $1 / 4$ or less supersymmetries.

\section{D-branes of PP-wave Strings Revisited}

In this section we will discuss the classification of D-branes in the pp-wave background. In the work [14], the allowed 1/2 supersymmetric D-brane configurations have been already classified for the case that the light-cone directions $(+,-)$ satisfy the Neumann conditions, by using the same method in this paper. Here we extend the discussion given in [14] by including the case that $(+,-)$-directions are the Dirichlet directions. In particular, we find the $1 / 2$ supersymmetric D-instanton configurations at and outside the origin.

By taking the Penrose limit [35] around a certain null geodesic in the $\operatorname{Ad}_{5} \times S^{5}$ background, we obtain the maximally supersymmetric IIB pp-wave background:

$$
\begin{aligned}
& d s^{2}=2 d X^{+} d X^{-}-\mu^{2}\left(X_{1}^{2}+\cdots+X_{8}^{2}\right)\left(d X^{-}\right)^{2}+\sum_{m=1}^{8}\left(d X^{m}\right)^{2}, \\
& F_{+1234}=F_{+5678}=4 \mu
\end{aligned}
$$

where $F_{A_{1} \cdots A_{5}}$ is a constant Ramond-Ramond self-dual five-form flux.

Our strategy is almost the same as in the AdS case. We define the projection operator (3.3) 
with the gluing matrix

$$
\begin{aligned}
& M=\left\{\begin{array}{l}
m \otimes i \sigma_{2}, \quad d=2(\bmod 4) \quad p=-1,3,7 \\
m \otimes \rho, \quad d=4(\bmod 4) \quad p=1,5,9
\end{array},\right. \\
& m=s \underline{\underline{A}}_{1} \ldots \Gamma^{\underline{A}_{d}}, \quad s=\left\{\begin{array}{cc}
1 & \text { for } X^{+}, X^{-}: \text {Neumann } \\
i & \text { for } X^{+}, X^{-} \text {: Dirichlet }
\end{array}, \quad \rho=\left\{\begin{array}{c}
\sigma_{1} \text { when } \sigma=\sigma_{3} \\
\sigma_{3} \text { when } \sigma=\sigma_{1}
\end{array} .\right.\right.
\end{aligned}
$$

We examine the $\kappa$-variation surface terms of the action (2.1) in the pp-wave background. As before, it turns out to be enough to consider the Wess-Zumino term (2.3). The supervielbein and the spin connection are given in Appendix C. We again divide the Wess-Zumino action into the three individual parts as follows:

$$
\begin{aligned}
& S_{\mathrm{WZ}}=S_{\mathrm{WZ}}^{\mu}+S_{\mathrm{WZ}}^{\mathcal{M}}+S_{\mathrm{WZ}}^{\mathrm{spin}}, \\
& S_{\mathrm{WZ}}^{\mu}=-2 i \int_{\Sigma}\left[\frac{1}{2} e^{A} \bar{\theta} \Gamma_{A} \sigma\left(d \theta+e^{-} \frac{\mu}{2}(f+g) i \sigma_{2} \theta+e^{m} \frac{\mu}{2} \widehat{\Gamma}_{m} i \sigma_{2} \theta\right)\right. \\
& +\frac{i}{8} \bar{\theta} \Gamma^{A}\left(d \theta+e^{-\frac{\mu}{2}}(f+g) i \sigma_{2} \theta+e^{m} \frac{\mu}{2} \widehat{\Gamma}_{m} i \sigma_{2} \theta\right) \\
& \left.\times \bar{\theta} \Gamma_{A} \sigma\left(d \theta+e^{-\frac{\mu}{2}}(f+g) i \sigma_{2} \theta+e^{m} \frac{\mu}{2} \widehat{\Gamma}_{m} i \sigma_{2} \theta\right)\right], \\
& S_{\mathrm{WZ}}^{\mathcal{M}}=-2 i \int_{\Sigma}\left[\frac{1}{24} e^{A} \bar{\theta} \Gamma_{A} \sigma \mathcal{M}^{2} D \theta\right] \\
& S_{\mathrm{WZ}}^{\mathrm{spin}}=-2 i \int_{\Sigma}\left[\frac{1}{2} e^{A} \bar{\theta} \Gamma_{A} \sigma\left(e_{*}^{m} \frac{\mu}{2} \Gamma_{m} \Gamma_{+} \theta\right)\right. \\
& +\frac{i}{8} \bar{\theta} \Gamma^{A}\left(d \theta+e^{-} \frac{\mu}{2}(f+g) i \sigma_{2} \theta+e^{m} \frac{\mu}{2} \widehat{\Gamma}_{m} i \sigma_{2} \theta\right) \cdot \bar{\theta} \Gamma_{A} \sigma\left(e_{*}^{m} \frac{\mu}{2} \Gamma_{m} \Gamma_{+} \theta\right) \\
& +\frac{i}{8} \bar{\theta} \Gamma^{A}\left(e_{*}^{m} \frac{\mu}{2} \Gamma_{m} \Gamma_{+} \theta\right) \cdot \bar{\theta} \Gamma_{A} \sigma\left(d \theta+e^{-} \frac{\mu}{2}(f+g) i \sigma_{2} \theta+e^{m} \frac{\mu}{2} \widehat{\Gamma}_{m} i \sigma_{2} \theta\right) \\
& \left.+\frac{i}{8} \bar{\theta} \Gamma^{A}\left(e_{*}^{m} \frac{\mu}{2} \Gamma_{m} \Gamma_{+} \theta\right) \cdot \bar{\theta} \Gamma_{A} \sigma\left(e_{*}^{m} \frac{\mu}{2} \Gamma_{m} \Gamma_{+} \theta\right)\right] \text {. }
\end{aligned}
$$

We examine the surface terms under the $\kappa$-variation of these three parts in turn. The vanishing conditions of the $\kappa$-variation surface terms coming from $S_{\mathrm{WZ}}^{\mu}$ lead to the classification of possible branes sitting at the origin. The vanishing conditions of the $\kappa$-variation surface terms of $S_{\mathrm{WZ}}^{\text {spin }}$ do not affect the classification at the origin, but lead to the additional conditions for the brane configurations outside the origin. The surface terms which come from $S_{\mathrm{WZ}}^{\mathcal{M}}$ vanish for the obtained configurations at and outside the origin. 
The $\kappa$-variation is defined by $\delta_{\kappa} E^{A}=0$, which means in this background

$$
\delta_{\kappa} X^{M}=-\frac{i}{2} \bar{\theta} \Gamma^{M} \delta_{\kappa} \theta+\mathcal{O}\left(\theta^{4}\right) .
$$

When we perform the $\kappa$-variation on $S_{\mathrm{WZ}}^{\mu}$, the surface terms are obtained as

$$
\begin{aligned}
\delta_{\kappa} S_{\mathrm{WZ}}^{\mu}=-2 i \int_{\partial \Sigma} & {\left[\frac{1}{2} \bar{\theta} \Gamma_{A} \sigma \delta_{\kappa} \theta d X^{M} e_{M}^{A}+\frac{i}{8}\left(\bar{\theta} \Gamma^{A} \delta_{\kappa} \theta \cdot \bar{\theta} \Gamma_{A} \sigma+\bar{\theta} \Gamma_{A} \sigma \delta_{\kappa} \theta \cdot \bar{\theta} \Gamma^{A}\right) d \theta\right.} \\
& -\frac{\mu i}{8} \bar{\theta} \Gamma^{-} \delta_{\kappa} \theta \cdot \bar{\theta} \Gamma_{A} \sigma(f+g) i \sigma_{2} \theta d X^{M} e_{M}^{A}-\frac{\mu i}{8} \bar{\theta} \Gamma^{m} \delta_{\kappa} \theta \cdot \bar{\theta} \sigma \widehat{\Gamma}_{m} i \sigma_{2} \theta d X^{M} e_{M}^{A} \\
& -\frac{\mu i}{16} \bar{\theta} \Gamma^{A} \delta_{\kappa} \theta \cdot \bar{\theta} \Gamma_{A} \sigma\left((f+g) i \sigma_{2} \theta d X^{M} e_{M}^{-}+\widehat{\Gamma}_{m} i \sigma_{2} \theta d X^{M} e_{M}^{m}\right) \\
& \left.+\frac{\mu i}{16} \bar{\theta} \Gamma^{A} \sigma \delta_{\kappa} \theta \cdot \bar{\theta} \Gamma_{A}\left((f+g) i \sigma_{2} \theta d X^{M} e_{M}^{-}+\widehat{\Gamma}_{m} i \sigma_{2} \theta d X^{M} e_{M}^{m}\right)\right] .
\end{aligned}
$$

The vanishing conditions for the first line read

$$
\bar{\theta} \Gamma^{\bar{A}} \sigma \delta_{\kappa} \theta=0 \quad \text { and } \quad \bar{\theta} \Gamma \underline{A} \delta_{\kappa} \theta=0
$$

which are satisfied by the projector given above. We thus have rederived the well-known condition, $p=$ odd, for IIB D $p$-branes in flat background. The additional conditions which come from the second, third and fourth lines are

$$
\bar{\theta} \Gamma_{\bar{A}}(f+g) \sigma i \sigma_{2} \theta=\bar{\theta} \Gamma_{\underline{A}}(f+g) i \sigma_{2} \theta=0 \quad \text { or } \quad-\in \text { Dirichlet },
$$

and

$$
\bar{\theta} \Gamma_{\bar{A}} \widehat{\Gamma}_{\bar{m}} \sigma i \sigma_{2} \theta=\bar{\theta} \Gamma_{\underline{A}} \widehat{\Gamma}_{\bar{m}} i \sigma_{2} \theta=0 .
$$

The condition (4.11) is satisfied for $p=3$ (1) $\bmod 4$ cases by one of the followings

- The number of Dirichlet directions in $\left(X^{1}, X^{2}, X^{3}, X^{4}\right)$ is even (odd), and the same condition is also satisfied for $\left(X^{5}, X^{6}, X^{7}, X^{8}\right)$,

- $-\in$ Dirichlet,

while the second condition (4.12) is satisfied if

- The number of Dirichlet directions in $\left(X^{+}, X^{1}, X^{2}, X^{3}, X^{4}\right)$ is even (odd), and the same condition is also satisfied for $\left(X^{+}, X^{5}, X^{6}, X^{7}, X^{8}\right)$. 
From these conditions, one can classify the possible branes in the pp-wave background. We denote branes with the world-volume extending along $m$ directions in $(1,2,3,4)$ and $n$ directions in $(5,6,7,8)$ as $(m, n)$-branes. In addition, when the directions $(+,-)$ are also Neumann directions, we denote as $(+,-; m, n)$-branes. We summarize in Tab. 2 the classification of $1 / 2$ supersymmetric D-branes sitting at the origin.

\begin{tabular}{|c|c|c|c|c|c|}
\hline D-instanton & D-string & D3-brane & D5-brane & D7-brane & D9-brane \\
\hline \hline & & $(+,-; 0,2)$, & $(+,-; 1,3)$, & $(+,-; 2,4)$, & \\
& & $(+,-; 2,0)$, & $(+,-; 3,1)$, & $(+,-; 4,2)$, & absent \\
& & & & \\
\hline
\end{tabular}

Table 2: The possible 1/2 supersymmetric configurations of D-branes sitting at the origin in the pp-wave background.

Next we examine the surface terms of the $\kappa$-variation of $S_{\mathrm{WZ}}^{\mathrm{spin}}$. We show that the surface terms vanish for branes sitting at the origin, but lead to additional conditions for branes sitting outside the origin. The $\kappa$-variation surface terms are

$$
\begin{aligned}
\delta_{\kappa} S_{\mathrm{WZ}}^{\mathrm{spin}}=-\frac{\mu^{2}}{8} & \int_{\partial \Sigma}\left[\bar{\theta} \Gamma^{\bar{A}} \delta_{\kappa} \theta \cdot \bar{\theta} \Gamma_{\bar{A}} \sigma \Gamma_{m} \Gamma_{+} \theta d X^{-}+2 \bar{\theta} \Gamma^{-} \delta_{\kappa} \theta \cdot \bar{\theta} \Gamma_{\bar{A}} \sigma \Gamma_{m} \Gamma_{+} \theta d X^{M} e_{M}^{\bar{A}}\right. \\
& \left.-\bar{\theta} \Gamma_{\underline{A}} \sigma \delta_{\kappa} \theta \cdot \bar{\theta} \Gamma^{A} \Gamma_{m} \Gamma_{+} \theta d X^{-}\right] X^{m}
\end{aligned}
$$

which vanish when $-\in$ Dirichlet or $X \underline{\underline{m}}=0$. Thus we found that for branes sitting at the origin the surface terms vanish, while for branes sitting outside the origin --direction has to be a Dirichlet direction. We summarize in Tab. 3 the possible branes sitting outside the origin.

\begin{tabular}{|c|c|c|c|c|c|}
\hline D-instanton & D-string & D3-brane & D5-brane & D7-brane & D9-brane \\
\hline \hline$(0,0)$ & $(0,2),(2,0)$ & $(1,3),(3,1)$ & $(2,4),(4,2)$ & absent & absent \\
\hline
\end{tabular}

Table 3: The possible 1/2 supersymmetric configurations of D-branes sitting outside the origin in the pp-wave background.

Finally we examine the $\kappa$-variation surface terms of $S_{\mathrm{WZ}}^{\mathcal{M}}$ and show that the surface terms 
vanish for the brane configurations classified above. The surface terms become

$$
\begin{aligned}
\delta_{\kappa} S_{\mathrm{WZ}}^{\mathcal{M}}=-\frac{\mu}{24} & \int_{\partial \Sigma}\left[\bar{\theta} \Gamma_{\bar{A}} \sigma(f+g) i \sigma_{2} \theta \cdot \bar{\theta} \Gamma^{-} \delta_{\kappa} \theta+\bar{\theta} \Gamma_{\bar{A}} \sigma \widehat{\Gamma}_{\bar{m}} i \sigma_{2} \theta \cdot \bar{\theta} \Gamma^{\bar{m}} \delta_{\kappa} \theta\right. \\
& +\bar{\theta} \Gamma_{\bar{A}} \sigma \Gamma_{i} \Gamma_{+} \theta \cdot \bar{\theta} \Gamma^{i} f i \sigma_{2} \delta_{\kappa} \theta-\bar{\theta} \Gamma_{\bar{A}} \sigma \Gamma_{i^{\prime}} \Gamma_{+} \theta \cdot \bar{\theta} \Gamma^{i^{\prime}} g i \sigma_{2} \delta_{\kappa} \theta \\
& \left.-\bar{\theta} \Gamma_{\bar{A}} \sigma \Gamma_{m n} \theta \cdot \bar{\theta} \widehat{\Gamma}^{m n} i \sigma_{2} \delta_{\kappa} \theta\right] d X^{M} e_{M}^{\bar{A}}
\end{aligned}
$$

One can show that these surface terms vanish when one of the followings is satisfied for $p=3$ (1) mod 4 cases,

- $-\in$ Dirichlet, and both $\left(X^{1}, X^{2}, X^{3}, X^{4}\right)$ and $\left(X^{5}, X^{6}, X^{7}, X^{8}\right)$ contain odd (even) number of Dirichlet directions

- - $\in$ Neumann, and both $\left(X^{1}, X^{2}, X^{3}, X^{4}\right)$ and $\left(X^{5}, X^{6}, X^{7}, X^{8}\right)$ contain even (odd) number of Dirichlet directions.

These conditions are satisfied by the brane configurations classified above.

The longitudinal D-brane configurations agree with those found in $[6,14]$. We found that there exist transverse D-branes sitting at and outside the origin. D-instantons preserving half of supersymmetries are allowed to freely sit in the pp-wave as in flat space and $\operatorname{AdS}_{5} \times S^{5}$. D-instanton correspond to a point in the pp-wave spacetime while the existence of $1 / 2$ supersymmetric D-instantons sitting outside the origin seems to be consistent to the homogeneity of the pp-wave geometry.

\section{Penrose Limits of D-branes of AdS String}

In this section we will consider Penrose limits [35] of the possible D-brane configurations in the AdS background obtained in section 3, and show that all of the D-branes in the pp-wave obtained in section 4 is recovered as Penrose limits of those in the AdS background.

The Penrose limit is taken as follows. One makes a set of light-cone coordinate $\left(X^{+}, X^{-}\right)$ from $\left(X^{0}, X^{9}\right)$, ${ }^{\boldsymbol{I}}$ scales $X^{+}$as $X^{+} \rightarrow \Omega^{2} X^{+}$and then take the limit $\Omega \rightarrow 0$. We distinguish the

\footnotetext{
IIf we make a pair of light-cone coordinates only in the AdS space or sphere, then flat spacetime is obtained as discussed in [37]. That is, in order to obtain the pp-wave background, we need to construct the lightcone coordinates by choosing one direction from the AdS coordinates and the other direction from the sphere coordinates.
} 
cases depending on the boundary conditions of the light-cone coordinate $\left(X^{+}, X^{-}\right)$as $(N, N)$ for $X^{ \pm} \in$ Neumann directions, and so on. For the fermionic coordinates, we scale $\theta_{+} \rightarrow \Omega \theta_{+}$ and take the limit $\Omega \rightarrow 0$. Some relevant aspects of the Penrose limit are explained in Appendix D.

We examine Penrose limits of $(\mathrm{N}, \mathrm{N})$ - and $(\mathrm{D}, \mathrm{D})$-cases.

\section{Penrose Limit of D9-brane}

There are no allowed 1/2 supersymmetric D9-brane configurations both in the $\mathrm{AdS}_{5} \times S^{5}$ and in the pp-wave. Hence, if we want to consider the correspondence of D9-branes in the AdS and those in the pp-wave, then we need to consider the less supersymmetric configurations of D9-branes.

\section{Penrose Limit of Dr-brane}

The possible D7-brane configurations are $(3,5)$ - and (5,3)-branes. Now we cannot consider (D,D)-case since there is no Dirichlet direction in the $S^{5}$ and $\mathrm{AdS}_{5}$, respectively.

First, we consider the Penrose limit of the $(3,5)$-branes, whose boundary condition is $s \Gamma^{a b} \otimes$ $i \sigma_{2} \theta=\theta$. In the $(\mathrm{N}, \mathrm{N})$-case the resulting boundary condition after the Penrose limit is given by

$$
s \Gamma^{i j} \otimes i \sigma_{2} \theta=\theta .
$$

That is, this configuration describes the $(+,-; 2,4)$-type D7-branes, which is included in the list of the allowed D-branes in the pp-wave case.

In the same way as in the (3,5)-brane case, we can investigate the Penrose limit of $(5,3)$ branes. The boundary condition after the Penrose limit is

$$
s \Gamma^{i^{\prime} j^{\prime}} \otimes i \sigma_{2} \theta=\theta
$$

which is the $(+,-; 4,2)$-brane boundary condition in the pp-wave. Thus, two types of D7brane configurations in the pp-wave are rederived as the Penrose limit of D7-branes in the AdS background.

\section{Penrose Limit of D5-brane}

The allowed D5-brane configurations are (2,4)- and (4,2)-branes. 
First, we consider $(2,4)$-type D5-branes whose boundary condition is $s \Gamma^{a_{1} a_{2} a_{3} a^{\prime}} \otimes \rho \theta=\theta$ with $\rho=\sigma_{1}$. When we choose the $(\mathrm{N}, \mathrm{N})$-boundary condition, the above condition becomes

$$
\Gamma^{i_{1} i_{2} i_{3}{ }^{\prime}} \otimes \rho \theta=\theta,
$$

after the Penrose limit. That is, we obtain $(+,-; 1,3)$-type D5-brane in the pp-wave. If we take the $(\mathrm{D}, \mathrm{D})$-condition, then we get the boundary condition:

$$
i \Gamma^{+-i j} \otimes \rho \theta=\theta,
$$

and hence $(2,4)$-type D5-branes are obtained. The resulting $(+,-; 1,3)$ - and $(2,4)$-type D5branes are possible in the pp-wave case.

Secondly, we study $(4,2)$-type D5-branes. In this case the boundary condition is given by

$s \Gamma^{a a_{1}^{\prime} a_{2}^{\prime} a_{3}^{\prime}} \otimes \rho \theta=\theta$. After taking the Penrose limit, we obtain, according to the choice of light-cone coordinates, the conditions:

$$
\begin{array}{ll}
\Gamma^{i i_{1}^{\prime} i_{2}^{\prime} i_{3}^{\prime}} \otimes \rho \theta=\theta & \text { for }(\mathrm{N}, \mathrm{N}) \text {-case } \\
i \Gamma^{+-i^{\prime} j^{\prime}} \otimes \rho \theta=\theta & \text { for }(\mathrm{D}, \mathrm{D}) \text {-case }
\end{array}
$$

For $(\mathrm{N}, \mathrm{N})$ - and $(\mathrm{D}, \mathrm{D})$-cases, we obtain $(+,-; 3,1)$ - and (4,2)-type branes, respectively. These configurations are also allowed in the pp-wave.

In the above discussion we have assumed the fundamental string case. It is also possible to consider the D-string by taking $\rho=\sigma_{3}$, and simultaneously D5-branes are replaced with NS5-branes. The only difference in our consideration of the Penrose limit is only a choice of $\sigma$ and the discussion in the D-string case is exactly the same as the above one.

\section{Penrose Limit of D3-brane}

The possible D3-brane configurations are (1,3)- and (3,1)-branes.

The boundary condition of (1,3)-type D3-brane is given by $s \Gamma^{a_{1} \cdots a_{4} a^{\prime} b^{\prime}} \otimes i \sigma_{2} \theta=\theta$, which becomes, according to the choice of the light-cone coordinates,

$$
\begin{array}{ll}
\Gamma^{i_{1} \cdots i_{4} i^{\prime} j^{\prime}} \otimes i \sigma_{2} \theta=\theta & \text { for (N,N)-case } \\
i \Gamma^{+-i_{1} i_{2} i_{3} i^{\prime}} \otimes i \sigma_{2} \theta=\theta & \text { for (D,D)-case. }
\end{array}
$$

When we consider the (3,1)-type D3-brane whose boundary condition is $s \Gamma^{a b a_{1}^{\prime} \cdots a_{4}^{\prime}} \otimes i \sigma_{2} \theta=\theta$, 
the resulting conditions after taking the Penrose limit are given by

$$
\begin{array}{lr}
\Gamma^{i j i_{1}^{\prime} \cdots i_{4}^{\prime}} \otimes i \sigma_{2} \theta=\theta & \text { for }(\mathrm{N}, \mathrm{N}) \text {-case } \\
i \Gamma^{+-i i_{1}^{\prime} i_{2}^{\prime} i_{3}^{\prime}} \otimes i \sigma_{2} \theta=\theta & \text { for }(\mathrm{D}, \mathrm{D}) \text {-case }
\end{array}
$$

Thus, we have obtained the D3-branes in the pp-wave as Penrose limits of D3-branes in the $\mathrm{AdS}_{5} \times S^{5}$ background.

\section{Penrose Limit of D1-brane}

We can take $(0,2)$ - and (2,0)-branes as possible D1-brane configurations. In this case we cannot take $(\mathrm{N}, \mathrm{N})$-type boundary condition.

The boundary conditions for the (0,2)- and (2,0)-type D-strings are

$$
s \Gamma^{a_{1} \cdots a_{5} a^{\prime} b^{\prime} c^{\prime}} \otimes \rho \theta=\theta \quad \text { and } \quad s \Gamma^{a_{1} a_{2} a_{3} a_{1}^{\prime} \cdots a_{5}^{\prime}} \otimes \rho \theta=\theta,
$$

with $\rho=\sigma_{1}$, respectively. When we consider the (D,D)-type boundary condition, these conditions become, after taking the Penrose limit,

$$
i \Gamma^{+-i_{1} \cdots i_{4} i^{\prime} j^{\prime}} \otimes \rho \theta=\theta \quad \text { and } \quad i \Gamma^{+-i j i_{1}^{\prime} \cdots i_{4}^{\prime}} \otimes \rho \theta=\theta
$$

and these conditions imply (0,2)- and (2,0)-type D-string configurations in the pp-wave, respectively. If we take $\rho=\sigma_{3}$, then (0,2)- and (2,0)-type F-string configurations in the pp-wave are obtained.

\section{Penrose Limit of D-instanton}

In this case we can consider the (D,D)-type boundary condition only. The condition for Dinstanton in the $\mathrm{AdS}_{5} \times S^{5}$ background is $i \Gamma^{01 \cdots 9} \otimes i \sigma_{2} \theta=\theta$. After taking the Penrose limit, we obtain the following condition:

$$
i \Gamma^{+-1 \cdots 8} \otimes i \sigma_{2} \theta=\theta
$$

This condition is nothing but that of D-instanton in the pp-wave.

Finally, we summarize the results obtained above in Tab. 4. 


\begin{tabular}{|c|c|c|}
\hline \multicolumn{3}{|c|}{ D7-branes } \\
\hline AdS & $(3,5)$ & $(5,3)$ \\
\hline$\downarrow$ Penrose & $D^{2} \swarrow \quad \searrow N^{2}$ & $D^{2} \swarrow$ \\
\hline pp-wave & $-\quad(+,-; 2,4)$ & $(+,-; 4,2)$ \\
\hline \multicolumn{3}{|c|}{ D5-branes } \\
\hline AdS & $(2,4)$ & $(4,2)$ \\
\hline$\downarrow$ Penrose & $D^{2} \swarrow \quad \searrow N^{2}$ & $D^{2} \swarrow \quad \searrow N^{2}$ \\
\hline pp-wave & $(2,4) \quad(+,-; 1,3)$ & $(4,2) \quad(+,-; 3,1)$ \\
\hline \multicolumn{3}{|c|}{ D3-branes } \\
\hline AdS & $(1,3)$ & $(3,1)$ \\
\hline$\downarrow$ Penrose & $D^{2} \swarrow \quad \searrow N^{2}$ & $\searrow N^{2}$ \\
\hline pp-wave & $(1,3) \quad(+,-; 0,2)$ & $(3,1) \quad(+,-; 2,0)$ \\
\hline \multicolumn{3}{|c|}{ D1-branes } \\
\hline $\mathrm{AdS}$ & $(0,2)$ & $(2,0)$ \\
\hline$\downarrow$ Penrose & $\searrow N^{2}$ & $D^{2} \swarrow$ \\
\hline pp-wave & $(0,2)$ & $(2,0)$ \\
\hline
\end{tabular}

Table 4: Penrose limit of D-branes of AdS string. 
We also briefly comment on $(\mathrm{N}, \mathrm{D})$ - or $(\mathrm{D}, \mathrm{N})$-cases. If we consider the Penrose limit in these cases, the boundary condition becomes $\theta_{-}=0$ for every D-brane. This condition implies the two types of conditions as follows:

$$
M=\Gamma^{+-} \otimes \mathbb{I}_{2} \quad \text { or } \quad M=\Gamma^{1 \cdots 8} \otimes \mathbb{I}_{2} .
$$

However, the boundary conditions associated with these gluing matrices do not eliminate the $\kappa$-variation surface terms. In fact, these matrices are not included in (4.3). It may be the case that we may not take $(\mathrm{N}, \mathrm{D})$ and $(\mathrm{D}, \mathrm{N})$-boundary conditions.

\section{Penrose Limit of $1 / 4$ Supersymmetric D-string}

Here we will consider the Penrose limit of D-string configuration preserving quarter of supersymmetries. This D-string is a (1,1)-type D-string. When we consider the $(\mathrm{N}, \mathrm{N})$-case, we obtain the $(+,-; 0,0)$-type $\mathrm{D}$-string in the pp-wave. This D-string configuration is nothing but the D-string found in the work [14]. If we take the (D,D)-boundary condition, we obtain the (1,1)-type D-string which is expected to be a $1 / 4$ supersymmetric D-string in the pp-wave.

\section{Conclusion and Discussion}

We have classified possible configurations of D-branes of a superstring in the $\operatorname{AdS}_{5} \times S^{5}$ background. In contrast to the pp-wave case, the D-string configuration is realized as a usual $1 / 2$ supersymmetric configuration. Notably, our classification result agrees with that of AdS brane configurations of the brane probe analysis. We have also extended the classification of allowed 1/2 supersymmetric D-branes in the pp-wave [14] by including the case that both of light-cone directions satisfy the Dirichlet conditions. In particular, we have found that $1 / 2$ supersymmetric D-instantons can exist at and outside the origin in both AdS and pp-wave backgrounds. The fact that D-instanton can survive even outside the origin seems to reflect the homogeneity of these backgrounds. In addition, we have investigated Penrose limits of the allowed D-branes in the $\operatorname{AdS}_{5} \times S^{5}$ background, and it has been shown that possible 1/2 supersymmetric configurations in the pp-wave case can be recovered as Penrose limits of those in the $\mathrm{AdS}_{5} \times S^{5}$.

We have mainly examined the standard configurations preserving half of supersymmetries, but it is interesting to approach $1 / 4$ or less supersymmetric D-branes of AdS string in our 
covariant formulation. In fact, as an example, we have presented a $1 / 4$ supersymmetric Dstring configuration in the $\mathrm{AdS}_{5} \times S^{5}$ background. This configuration has been shown to reduce to the $1 / 4$ supersymmetric D-string in the pp-wave preserving 8 dynamical supersymmetries. D-branes of this type are called $D_{+}$-branes in the works [8]. On the other hand, the standard D-branes are called $D_{-}$-branes. In this paper we have clarified the AdS origin of $D_{-}$-branes. It may be possible to find the AdS origin of $D_{+}$-branes. Of course, we can also seek for the AdS origin of oblique D-branes, curved D-branes and intersections of D-branes in the pp-wave case (For a work in this direction, [44]). In addition, giant gravitons" or baryon vertex operators may be investigated in our covariant formulation if we treat these objects in the $\operatorname{AdS}_{5} \times S^{5}$ by the use of our formulation. It is also interesting to examine Dirichlet boundaries of an open D3-brane constructed in [46] and compare the result with that obtained here. Another direction is to study D-branes on other AdS backgrounds such as $\mathrm{AdS}_{5} \times S^{5} / \mathbb{Z}_{N}$ and $\mathrm{AdS}_{5} \times T^{1,1}[47]$. We will report all of these problems in another place in the near future [48].

It is also an interesting problem to apply our results to defect conformal field theories via the AdS/CFT correspondence [49]. Recall that a stringy nature in pp-wave string theories played an important role in studies of the AdS/CFT correspondence at the stringy level beyond supergravity approximation [27]. Our classification results and our methods are expected to promote the understanding of AdS/CFT correspondence.

\section{Acknowledgments}

We would like to thank Machiko Hatsuda and Katsuyuki Sugiyama for useful discussion.

\section{Appendix}

\section{A Notation and Convention}

In this place we will summarize miscellaneous notation and convention used in this paper.

\footnotetext{
${ }^{\|}$The matrix model on the pp-wave also has supersymmetric fuzzy sphere solutions (giant graviton) [27]. Giant gravitons in the pp-wave matrix model are discussed in [29,45].
} 


\section{Notation of Coordinates}

For the superstring in the ten-dimensional curved space-time: $\operatorname{AdS}_{5} \times S^{5}$ and pp-wave backgrounds, we use the following notation of supercoordinates for its superspace $\left(X^{M}, \theta^{\bar{\alpha}}\right)$ :

$$
M=\left(\mu, \mu^{\prime}\right), \quad \mu=(0,1,2,3,4) \in A d S_{5}, \quad \mu^{\prime}=(5,6,7,8,9) \in S^{5}
$$

and the background metric is expressed by $G_{M N}$. The coordinates in the Lorentz frame are denoted by $\left(X^{A}, \theta^{\alpha}\right)$ :

$$
\begin{aligned}
& A=\left(a, a^{\prime}\right), \quad a=0,1,2,3,4, \quad a^{\prime}=5,6,7,8,9 \quad \text { for AdS case, } \\
& A=\left(+,-, i, i^{\prime}\right), \quad i=1,2,3,4, \quad i^{\prime}=5,6,7,8, \quad m=\left(i, i^{\prime}\right) \quad \text { for pp-wave case, }
\end{aligned}
$$

and its metric is described by $\eta_{A B}=\operatorname{diag}(-1,+1, \ldots,+1)$ with $\eta_{00}=-1$. The light-cone coordinates are defined by $X^{ \pm} \equiv \frac{1}{\sqrt{2}}\left(X^{9} \pm X^{0}\right)$. The coordinates of world-sheet are parameterized by $\left(\sigma^{1}, \sigma^{2}\right)=(\tau, \sigma)$. The induced metric on the world-sheet is represented by $g_{i j}(i, j=1,2)$.

\section{SO(1,9) Gamma Matrices}

We denote two 16-component Majorana-Weyl spinors as $\theta_{1}$ and $\theta_{2}$, and the $S O(1,9)$ Clifford algebra is written as

$$
\left\{\Gamma^{A}, \Gamma^{B}\right\}=2 \eta^{A B}, \quad\left\{\Gamma^{M}, \Gamma^{N}\right\}=2 G^{M N}, \quad \Gamma^{A} \equiv e_{M}^{A} \Gamma^{M}, \quad \Gamma^{M} \equiv e_{A}^{M} \Gamma^{A},
$$

where the light-cone components of the $S O(1,9)$ gamma matrices are

$$
\Gamma_{ \pm}=\frac{1}{\sqrt{2}}\left(\Gamma_{9} \pm \Gamma_{0}\right), \quad\left\{\Gamma^{+}, \Gamma^{-}\right\}=2 \mathbb{I}_{16}
$$

The chirality operator and the Dirac conjugate are defined as

$$
\Gamma^{11} \equiv \Gamma_{0 \cdots 9}, \quad \bar{\theta} \equiv \theta^{T} \mathcal{C},
$$

where the charge conjugation matrix $\mathcal{C}$ satisfies the relations:

$$
\mathcal{C}^{T}=-\mathcal{C}, \quad \mathcal{C}^{-1}\left(\Gamma^{M}\right)^{T} \mathcal{C}=-\Gamma^{M}
$$

In this paper we mainly use the 32 -component representation $\theta^{T}=\left(\theta_{1}, \theta_{2}\right)$. 


\section{B Coset Construction of Supervielbein in the $\operatorname{AdS}_{5} \times \mathbf{S}^{5}$}

Here we will briefly review the coset construction of supervielbein in the $\operatorname{AdS}_{5} \times S^{5}$ background. The super- $\mathrm{AdS}_{5} \times S^{5}$-algebra is regarded as $s u(2,2 \mid 4)$, and the $\operatorname{AdS}_{5} \times S^{5}$ manifold can be constructed as a coset $S U(2,2 \mid 4) /(S O(1,4) \times S O(5))$.

The bosonic part of the super $\operatorname{AdS}_{5} \times S^{5}$-algebra is $s o(2,4) \times s o(6)$ given by

$$
\begin{aligned}
& {\left[P_{a}, P_{b}\right]=\lambda^{2} M_{a b}, \quad\left[P_{a^{\prime}}, P_{b^{\prime}}\right]=-\lambda^{2} M_{a^{\prime} b^{\prime}},} \\
& {\left[P_{a}, M_{b c}\right]=\eta_{a b} P_{c}-\eta_{a c} P_{b}, \quad\left[P_{a^{\prime}}, M_{b^{\prime} c^{\prime}}\right]=\eta_{a^{\prime} b^{\prime}} P_{c^{\prime}}-\eta_{a^{\prime} c^{\prime}} P_{b^{\prime}},} \\
& {\left[M_{a b}, M_{c d}\right]=\eta_{b c} M_{a d}+3 \text { terms }, \quad\left[M_{a^{\prime} b^{\prime}}, M_{c^{\prime} d^{\prime}}\right]=\eta_{b^{\prime} c^{\prime}} M_{a^{\prime} d^{\prime}}+3 \text { terms . }}
\end{aligned}
$$

The parameter $\lambda$ characterizes the $\mathrm{AdS}_{5} \times S^{5}$ geometry, and if we take the limit $\lambda \rightarrow 0$ then the geometry reduces to the ten-dimensional Minkowski spacetime.

Now we decompose the $S O(1,9)$ gamma matrices $\Gamma^{A}$ 's as follows:

$$
\begin{aligned}
& \Gamma^{a}=\gamma^{a} \otimes \mathbb{I} \otimes \tau_{1} \quad(a=0,1,2,3,4), \\
& \Gamma^{a^{\prime}}=\mathbb{I} \otimes \gamma^{a^{\prime}} \otimes \tau_{2} \quad\left(a^{\prime}=5,6,7,8,9\right), \\
& \Gamma^{11}=\Gamma_{0} \cdots \Gamma_{9}=\mathbb{I} \otimes \mathbb{I} \otimes \tau_{3},
\end{aligned}
$$

where the $2 \times 2$ matrices $\tau_{i}$ 's are the standard Pauli matrices. The gamma matrices of the $\mathrm{AdS}_{5}$ part $\gamma^{a}$ 's are

$$
\gamma^{i}(i=0,1,2,3) \text { and } \gamma^{4} \equiv i \gamma_{0123}
$$

and hence $\left(\gamma^{4}\right)^{2}=1$ and $i \gamma_{01234}=+1$. For the gamma matrices of the $S^{5}$ part $\gamma^{a^{\prime}}$, s, we use

$$
\gamma^{i^{\prime}}\left(i^{\prime}=5,6,7,8\right) \text { and } \gamma^{9} \equiv \gamma_{5678}
$$

and then $\gamma^{56789}=+1$. The charge conjugation matrix $\mathcal{C}$ is defined as

$$
\mathcal{C} \equiv C \otimes C^{\prime} \otimes i \tau_{2}
$$

where $C$ and $C^{\prime}$ are the charge conjugation matrices in the $\mathrm{AdS}_{5}$ and $S^{5}$, respectively. We introduce chirality projection operators $h_{ \pm}=\frac{1}{2}\left(1 \pm \Gamma^{11}\right)$ and Majorana-Weyl supercharges $Q_{I}(I=1,2)$ by $Q_{I} h_{+}=Q_{I}$. By the use of these quantities, the fermionic part of the super$\mathrm{AdS}_{5} \times S^{5}$ algebra given in [50] is rewritten as

$$
\begin{aligned}
& \left\{Q_{I}, Q_{J}\right\}=2 i \mathcal{C} \Gamma^{A}(\mathbb{I})_{I J} h_{+} P_{A}+i \lambda \mathcal{C} \Gamma^{a b} \mathcal{I}\left(i \sigma_{2}\right)_{I J} h_{+} M_{a b}-i \lambda \mathcal{C} \Gamma^{a^{\prime} b^{\prime}} \mathcal{J}\left(i \sigma_{2}\right)_{I J} h_{+} M_{a^{\prime} b^{\prime}}, \\
& {\left[Q_{I}, P_{a}\right]=+\frac{\lambda}{2} Q_{J}\left(i \sigma_{2}\right)_{J I} \Gamma_{a} \mathcal{I}, \quad\left[Q_{I}, P_{a^{\prime}}\right]=-\frac{\lambda}{2} Q_{J}\left(i \sigma_{2}\right)_{J I} \Gamma_{a^{\prime}} \mathcal{J},} \\
& {\left[Q_{I}, M_{A B}\right]=-\frac{1}{2} Q_{I} \Gamma_{A B}, \quad \mathcal{I} \equiv \Gamma^{01234}, \quad \mathcal{J} \equiv \Gamma^{56789}}
\end{aligned}
$$


where the Pauli matrices $\left(\sigma_{i}\right)_{I J}$ act on the two-component supercharges $Q_{I}$.

Now we consider $G=g_{x} g_{\theta}$ defined as

$$
g_{x}=\mathrm{e}^{X^{a} P_{a}+X^{a^{\prime}} P_{a^{\prime}}}, \quad g_{\theta}=\mathrm{e}^{Q \theta}, \quad Q=\left(Q_{1}, Q_{2}\right), \quad \theta=\left(\begin{array}{c}
\theta_{1} \\
\theta_{2}
\end{array}\right) .
$$

The supervielbeins $E^{A}$ and $E^{\alpha}$, and super spin connection $E^{A B}$ are defined by the relations

$$
\begin{aligned}
G^{-1} d G & =E^{A} P_{A}+\frac{1}{2} E^{A B} M_{A B}+Q_{\alpha} E^{\alpha}, \\
g_{x}^{-1} d g_{x} & =e^{A} P_{A}+\frac{1}{2} \omega^{A B} M_{A B},
\end{aligned}
$$

where $e^{A}$ and $\omega^{A B}$ are the vielbein and the spin connection of the $\operatorname{AdS}_{5} \times S^{5}$. We can derive the expressions for the (super)vielbein and the (super)spin connection by expanding the l.h.s of the above relations. As a result, we obtain the following expressions

$$
\begin{aligned}
E^{A} & =e^{A}+i \bar{\theta} \Gamma^{A}\left(\frac{\sinh (\mathcal{M} / 2)}{\mathcal{M} / 2}\right)^{2} D \theta, \\
E^{\alpha} & =\left(\frac{\sinh \mathcal{M}}{\mathcal{M}} D \theta\right)^{\alpha}, \\
E^{A B} & =\omega^{A B}-i \lambda \widehat{\theta} \widehat{\Gamma}^{A B} i \sigma_{2}\left(\frac{\sinh (\mathcal{M} / 2)}{\mathcal{M} / 2}\right)^{2} D \theta,
\end{aligned}
$$

where we have introduced the quantities:

$$
\begin{aligned}
\mathcal{M}^{2} & =i \lambda\left(\widehat{\Gamma}_{A} i \sigma_{2} \theta \cdot \bar{\theta} \Gamma^{A}-\frac{1}{2} \Gamma_{A B} \theta \cdot \bar{\theta} \widehat{\Gamma}^{A B} i \sigma_{2}\right), \\
D \theta & =d \theta+\frac{\lambda}{2} e^{A} \widehat{\Gamma}_{A} i \sigma_{2} \theta+\frac{1}{4} \omega^{A B} \Gamma_{A B} \theta, \\
\widehat{\Gamma}_{A} & \equiv\left(-\Gamma_{a} \mathcal{I}, \Gamma_{a^{\prime}} \mathcal{J}\right), \quad \widehat{\Gamma}_{A B} \equiv\left(-\Gamma_{a b} \mathcal{I}, \Gamma_{a^{\prime} b^{\prime}} \mathcal{J}\right) .
\end{aligned}
$$

The vielbein and the spin connection of the $\operatorname{AdS}_{5} \times S^{5}$ background are given by

$$
\begin{aligned}
& e^{a}=\left(\frac{\sinh Y}{Y} d X\right)^{a}, \quad e^{a^{\prime}}=\left(\frac{\sinh Y^{\prime}}{Y^{\prime}} d X^{\prime}\right)^{a^{\prime}} \\
& \left.\omega^{a b}=-\lambda^{2} X^{[a}\left(\frac{\sinh (Y / 2)}{Y / 2} d X\right)^{2 b]}, \quad \omega^{a^{\prime} b^{\prime}}=+\lambda^{2} X^{\left[a^{\prime}\right.}\left(\frac{\sinh \left(Y^{\prime} / 2\right)}{Y^{\prime} / 2} d X\right)^{2} b^{\prime}\right] \\
& Y_{b}^{2 a}=\lambda^{2}\left(X^{2} \delta_{b}^{a}-X^{a} X_{b}\right), \quad Y_{b}^{\prime 2 a}=-\lambda^{2}\left(X^{\prime 2} \delta_{b^{\prime}}^{a^{\prime}}-X^{a^{\prime}} X_{b^{\prime}}\right) .
\end{aligned}
$$

The above expressions of the spin connection are used in considering the D-branes sitting outside the origin. 


\section{Coset Construction of Supervielbein in the PP-wave}

The super-pp-wave algebra is generated by momenta, $P_{-}$and $P_{m}$, boost, $P_{m}^{*}$, Lorentz generators of $S O(4) \times S O(4), M_{r s}$ and $M_{r^{\prime} s^{\prime}}$, and supercharge, $Q_{I}$, as

$$
\begin{aligned}
& {\left[P_{-}, P_{m}\right]=P_{m}^{*}, \quad\left[P_{-}, P_{m}^{*}\right]=-\mu^{2} P_{m}, \quad\left[P_{m}^{*}, P_{n}\right]=-\mu^{2} \delta_{m n} P_{+},} \\
& {\left[M_{m n}, P_{p}\right]=\delta_{n p} P_{m}-\delta_{m p} P_{n}, \quad\left[M_{m n}, P_{p}^{*}\right]=\delta_{n p} P_{m}^{*}-\delta_{m p} P_{n}^{*},} \\
& {\left[M_{m n}, M_{p q}\right]=\delta_{n p} M_{m q}+3 \text { terms },} \\
& {\left[P_{r}, Q_{I}\right]=-\frac{\mu}{2} Q_{J} \Gamma_{r} \Gamma_{+} f\left(i \sigma_{2}\right)_{J I}, \quad\left[P_{r^{\prime}}, Q_{I}\right]=\frac{\mu}{2} Q_{J} \Gamma_{r^{\prime}} \Gamma_{+} g\left(i \sigma_{2}\right)_{J I},} \\
& {\left[P_{-}, Q_{I}\right]=\frac{\mu}{2} Q_{J}(f+g)\left(i \sigma_{2}\right)_{J I}, \quad\left[P_{m}^{*}, Q_{I}\right]=\frac{\mu^{2}}{2} Q_{I} \Gamma_{m} \Gamma_{+}, \quad\left[M_{m n}, Q_{I}\right]=\frac{1}{2} Q_{I} \Gamma_{m n},} \\
& \left\{Q_{I}, Q_{J}\right\}=i \mathcal{C} \Gamma^{+} \mathbb{I}_{I J} h_{+} P_{+}+i \mathcal{C} \Gamma^{-} \mathbb{I}_{I J} h_{+} P_{-}+i \mathcal{C} \Gamma^{m} \mathbb{I}_{I J} h_{+} P_{m}+\frac{i}{\mu} \mathcal{C} \Gamma^{r} f\left(i \sigma_{2}\right)_{I J} h_{+} P_{r}^{*}, \\
& \quad-\frac{i}{\mu} \mathcal{C} \Gamma^{r^{\prime}} g\left(i \sigma_{2}\right)_{I J} h_{+} P_{r^{\prime}}^{*}+i \mu \mathcal{C} \Gamma^{r s} \Gamma_{+} f\left(i \sigma_{2}\right)_{I J} h_{+} M_{r s}-i \mu \mathcal{C} \Gamma^{r^{\prime} s^{\prime}} \Gamma_{+} g\left(i \sigma_{2}\right)_{I J} h_{+} M_{r^{\prime} s^{\prime}},
\end{aligned}
$$

where $f \equiv \Gamma^{1234}$ and $g \equiv \Gamma^{5678}$. It is known that this superalgebra is an Inonu-Wigner contraction of the super- $\mathrm{AdS}_{5} \times S^{5}$ algebra [51] $\left(\right.$ For $\mathrm{AdS}_{4 / 7} \times S^{7 / 4}$ cases, see [52]). We parameterize the group manifold by

$$
G=g_{x} g_{\theta}, \quad g_{x}=\mathrm{e}^{X^{+} P_{+}} \mathrm{e}^{X^{-} P_{-}} \mathrm{e}^{X^{m} P_{m}}, \quad g_{\theta}=\mathrm{e}^{Q_{I} \theta_{I}},
$$

and define Maurer-Cartan one-forms by

$$
G^{-1} d G=E^{+} P_{+}+E^{-} P_{-}+E^{m} P_{m}+\frac{1}{2} E^{m n} M_{m n}+E_{*}^{m} P_{m}^{*}+Q_{\alpha} E^{\alpha} .
$$

After simple algebraic calculation, one obtains the supervielbein described by

$$
E^{A}=e^{A}+\frac{i}{2} \bar{\theta} \Gamma^{A}\left(\frac{\sinh (\mathcal{M} / 2)}{\mathcal{M} / 2}\right)^{2} D \theta, \quad E^{\alpha}=\left(\frac{\sinh \mathcal{M}}{\mathcal{M}} D \theta\right)^{\alpha}
$$

where we have introduced several quantities:

$$
\begin{aligned}
\mathcal{M}^{2}= & i \frac{\mu}{2}(f+g) i \sigma_{2} \theta \cdot \bar{\theta} \Gamma^{-}+i \frac{\mu}{2} \widehat{\Gamma}_{m} i \sigma_{2} \theta \cdot \bar{\theta} \Gamma^{m}+i \frac{\mu}{2} \Gamma_{r} \Gamma_{+} \theta \cdot \bar{\theta} \Gamma^{r} f i \sigma_{2} \\
& -i \frac{\mu}{2} \Gamma_{r^{\prime}} \Gamma_{+} \theta \cdot \bar{\theta} \Gamma^{r^{\prime}} g i \sigma_{2}-i \frac{\mu}{2} \Gamma_{m n} \theta \cdot \bar{\theta} \widehat{\Gamma}^{m n} i \sigma_{2} \\
D \theta= & d \theta+e^{-} \frac{\mu}{2}(f+g) i \sigma_{2} \theta+e^{m} \frac{\mu}{2} \widehat{\Gamma}_{m} i \sigma_{2} \theta+e_{*}^{m} \frac{\mu^{2}}{2} \Gamma_{m} \Gamma_{+} \theta, \\
\widehat{\Gamma}_{m}= & \left(-\Gamma_{r} \Gamma_{+} f, \Gamma_{r^{\prime}} \Gamma_{+} g\right), \quad \widehat{\Gamma}_{m n}=\left(-\Gamma_{r s} \Gamma_{+} f, \Gamma_{r^{\prime} s^{\prime}} \Gamma_{+} g\right), \\
e^{+}= & d X^{+}-\frac{\mu^{2}}{2}\left(X^{m}\right)^{2} d X^{-}, \quad e^{-}=d X^{-}, \quad e^{m}=d X^{m}, \quad e_{*}^{m}=X^{m} d X^{-} .
\end{aligned}
$$


In this parameterization, the pp-wave metric becomes the standard form

$$
d s^{2}=2 e^{+} e^{-}+\left(e^{m}\right)^{2}=2 d X^{+} d X^{-}-\mu^{2}\left(X^{m}\right)^{2}\left(d X^{-}\right)^{2}+\left(d X^{m}\right)^{2} .
$$

The constructed supervielbein is used in section 4 for the classification of D-branes in the pp-wave background.

\section{Penrose Limit}

It is known that the pp-wave background is obtained via the Penrose limit from the $\operatorname{AdS}_{5} \times S^{5}$ background. The Penrose limit is taken by rescaling coordinates as

$$
X^{+} \rightarrow \Omega^{2} X^{+}, \quad X^{m} \rightarrow \Omega X^{m}, \quad \theta_{+} \rightarrow \Omega \theta_{+}
$$

where $\theta_{+}$is defined by $\theta_{+}=\mathcal{P}_{+} \theta$ with $\mathcal{P}_{+}=\frac{1}{2} \Gamma_{+} \Gamma_{-}$, and then by bringing $\Omega$ to zero. After the Penrose limit, the scale $\lambda$ of $\mathrm{AdS}_{5}$ or $S^{5}$ can be regarded as the scale $\mu$ of the pp-wave**. Noting that the super-pp-wave algebra is an Inonu-Wigner contraction of the super- $\mathrm{Ad}_{5} \times S^{5}$ algebra [51], the Penrose limit of the $\mathrm{AdS}_{5} \times S^{5}$ group manifold parameterized by $g_{x}=\mathrm{e}^{X^{a} P_{a}+X^{a^{\prime}} P_{a^{\prime}}}$ turns to be the pp-wave group manifold parameterized by $g_{x}=\mathrm{e}^{X^{+} P_{+}+X^{-} P_{-}+X^{m} P_{m}}$. In this parameterization, the metric is calculated to be

$$
\begin{aligned}
d s^{2} & =2 e^{+} e^{-}+\left(e^{m}\right)^{2}, \\
e^{+} & =d X^{+}+\left\{1-\frac{\sin \mu X^{-}}{\mu X^{-}}\right\} \frac{X_{m}}{\left(X^{-}\right)^{2}}\left(X^{-} d X^{m}-X^{m} d X^{-}\right), \\
e^{-} & =d X^{-}, \\
e^{m} & =\frac{\sin \mu X^{-}}{\mu X^{-}} d X^{m}+\left\{1-\frac{\sin \mu X^{-}}{\mu X^{-}}\right\} \frac{X^{m}}{X^{-}} d X^{-} .
\end{aligned}
$$

In order to make the metric to be of the standard form (4.1), we perform the coordinate transformation

$$
\hat{X}^{+}=X^{+}+\frac{\mu}{2} \frac{\mu X^{-}-\sin \mu X^{-} \cos \mu X^{-}}{\sin ^{2} \mu X^{-}}\left(\hat{X}^{m}\right)^{2}, \quad \hat{X}^{-}=X^{-}, \quad \hat{X}^{m}=\frac{\sin \mu X^{-}}{\mu X^{-}} X^{m},
$$

${ }^{* *}$ The scale $\lambda \sim 1 / R$ can be absorbed and the scale $\mu$ can be introduced by a field redefinition

$$
X^{+} \rightarrow \frac{\lambda^{2} \Omega^{2}}{\mu} X^{+}, \quad X^{-} \rightarrow \mu X^{-}, \quad X^{m} \rightarrow \lambda \Omega X^{m} .
$$

This reveals the fact that the Penrose limit $\Omega \rightarrow 0$ is equivalent to the limit $R \rightarrow \infty$ which is considered in the literature. 
under which the metric becomes the standard form

$$
d s^{2}=2 d \hat{X}^{+} d \hat{X}^{-}-\mu^{2}\left(\hat{X}^{m}\right)^{2}\left(d \hat{X}^{-}\right)^{2}+\left(d \hat{X}^{m}\right)^{2}
$$

This coordinate transformation reveals the fact that $X^{m}=0$ is mapped to $\hat{X}^{m}=0$ and $X^{+}=X^{-}=0$ corresponds to $\hat{X}^{+}=\hat{X}^{-}=0$.

\section{References}

[1] J. Polchinski, "Dirichlet-Branes and Ramond-Ramond Charges," Phys. Rev. Lett. 75 (1995) 4724 [arXiv:hep-th/9510017].

[2] T. Banks, W. Fischler, S. H. Shenker and L. Susskind, "M theory as a matrix model: A conjecture," Phys. Rev. D 55 (1997) 5112 [arXiv:hep-th/9610043].

[3] M. Blau, J. Figueroa-O'Farrill, C. Hull and G. Papadopoulos, "A new maximally supersymmetric background of IIB superstring theory," JHEP 0201 (2002) 047 [arXiv:hepth/0110242].

[4] R. R. Metsaev, "Type IIB Green-Schwarz superstring in plane wave Ramond-Ramond background," Nucl. Phys. B 625 (2002) 70 [arXiv:hep-th/0112044].

[5] R. R. Metsaev and A. A. Tseytlin, "Exactly solvable model of superstring in plane wave Ramond-Ramond background," Phys. Rev. D 65 (2002) 126004 [arXiv:hep-th/0202109].

[6] A. Dabholkar and S. Parvizi, "Dp branes in pp-wave background," Nucl. Phys. B 641 (2002) 223 [arXiv:hep-th/0203231].

[7] M. Billo and I. Pesando, "Boundary states for GS superstrings in an Hpp wave background," Phys. Lett. B 536 (2002) 121 [arXiv:hep-th/0203028].

[8] K. Skenderis and M. Taylor, "Branes in AdS and pp-wave spacetimes," JHEP 0206 (2002) 025 [arXive:hep-th/0204054]; "Open strings in the plane wave background. I: Quantization and symmetries," Nucl. Phys. B 665 (2003) 3 [arXiv:hep-th/0211011]; "Open strings in the plane wave background. II: Superalgebras and spectra," JHEP 0307 (2003) 006 [arXiv:hepth/0212184]; "An overview of branes in the plane wave background," Class. Quant. Grav. 
20 (2003) S567 [arXiv:hep-th/0301221]; D. Z. Freedman, K. Skenderis and M. Taylor, "Worldvolume supersymmetries for branes in plane waves," arXiv:hep-th/0306046.

[9] P. Bain, P. Meessen and M. Zamaklar, "Supergravity solutions for D-branes in Hpp-wave backgrounds," Class. Quant. Grav. 20 (2003) 913 [arXiv:hep-th/0205106].

[10] O. Bergman, M. R. Gaberdiel and M. B. Green, "D-brane interactions in type IIB planewave background," JHEP 0303 (2003) 002 [arXiv:hep-th/0205183]; M. R. Gaberdiel and M. B. Green, "The D-instanton and other supersymmetric D-branes in IIB plane-wave string theory," Annals Phys. 307 (2003) 147 [arXiv:hep-th/0211122]; M. R. Gaberdiel, M. B. Green, S. Schafer-Nameki and A. Sinha, "Oblique and curved D-branes in IIB plane-wave string theory," JHEP 0310 (2003) 052 [arXiv:hep-th/0306056].

[11] S. Seki, "D5-brane in Anti-de Sitter space and Penrose limit," Phys. Lett. B 542 (2002) 162 [Phys. Lett. B 542 (2002) 165] [arXiv:hep-th/0205266]; D. Mateos and S. Ng, "Penrose limits of the baryonic D5-brane," JHEP 0208 (2002) 005 [arXiv:hep-th/0205291].

[12] Y. Michishita, "D-branes in NSNS and RR pp-wave backgrounds and S-duality," JHEP 0210 (2002) 048 [arXiv:hep-th/0206131].

[13] K. Sugiyama and K. Yoshida, "Type IIA string and matrix string on pp-wave," Nucl. Phys. B 644 (2002) 128 [arXiv:hep-th/0208029]; S. Hyun and H. Shin, "N = (4,4) type IIA string theory on pp-wave background," JHEP 0210 (2002) 070 [arXiv:hep-th/0208074].

[14] P. Bain, K. Peeters and M. Zamaklar, "D-branes in a plane wave from covariant open strings," Phys. Rev. D 67 (2003) 066001 [arXiv:hep-th/0208038].

[15] H. Takayanagi and T. Takayanagi, "Notes on giant gravitons on pp-waves," JHEP 0212 (2002) 018 [arXiv:hep-th/0209160].

[16] S. Hyun and H. Shin, "Solvable N = $(4,4)$ type IIA string theory in plane-wave background and D-branes," Nucl. Phys. B 654 (2003) 114 [arXiv:hep-th/0210158].

[17] Y. Hikida and S. Yamaguchi, "D-branes in pp-waves and massive theories on worldsheet with boundary," JHEP 0301 (2003) 072 [arXiv:hep-th/0210262].

[18] R. R. Metsaev, "Supersymmetric D3 brane and N = 4 SYM actions in plane wave backgrounds," Nucl. Phys. B 655 (2003) 3 [arXiv:hep-th/0211178]. 
[19] S. Hyun, J. Park and H. Shin, "Covariant description of D-branes in IIA plane-wave background," Phys. Lett. B 559 (2003) 80 [arXiv:hep-th/0212343].

[20] H. Shin, K. Sugiyama and K. Yoshida, "Partition function and open/closed string duality in type IIA string theory on a pp-wave," Nucl. Phys. B 669 (2003) 78 [arXiv:hep-th/0306087]; Y. Kim and J. Park, "Boundary states in IIA plane-wave background," Phys. Lett. B 572 (2003) 81 [arXiv:hep-th/0306282].

[21] J. Kim, B. H. Lee and H. S. Yang, "Superstrings and D-branes in plane wave," Phys. Rev. D 68 (2003) 026004 [arXiv:hep-th/0302060]; K. S. Cha, B. H. Lee and H. S. Yang, "Intersecting D-branes in type IIB plane wave background," arXiv:hep-th/0307146; "A Complete Classification of D-branes in Type IIB Plane Wave Background," arXiv:hepth/0310177.

[22] C. S. Chu and P. M. Ho, "Noncommutative D-brane and open string in pp-wave background with B-field," Nucl. Phys. B 636 (2002) 141 [arXiv:hep-th/0203186]; A. Kumar, R. R. Nayak and Sanjay, "D-brane solutions in pp-wave background," Phys. Lett. B 541 (2002) 183 [arXiv:hep-th/0204025]; M. Alishahiha and A. Kumar, "D-brane solutions from new isometries of pp-waves," Phys. Lett. B 542 (2002) 130 [arXiv:hep-th/0205134]; M. Alishahiha, M. A. Ganjali, A. Ghodsi and S. Parvizi, "On type IIA string theory on the PP-wave background," Nucl. Phys. B 661 (2003) 174 [arXiv:hep-th/0207037]; A. Biswas, A. Kumar and K. L. Panigrahi, "p - p' branes in pp-wave background," Phys. Rev. D 66 (2002) 126002 [arXiv:hep-th/0208042];O. J. Ganor and U. Varadarajan, "Nonlocal effects on D-branes in plane-wave backgrounds," JHEP 0211 (2002) 051 [arXiv:hep-th/0210035]; M. Alishahiha and O. J. Ganor, "Twisted backgrounds, pp-waves and nonlocal field theories," JHEP 0303 (2003) 006 [arXiv:hep-th/0301080]; R. R. Nayak, "D-branes at angle in pp-wave background," Phys. Rev. D 67 (2003) 086006 [arXiv:hep-th/0210230]; L. F. Alday and M. Cirafici, "An example of localized D-branes solution on pp-wave backgrounds," JHEP 0305 (2003) 006 [arXiv:hep-th/0301253]; K. Ito and Y. Sekino, "Penrose limit and enhancon geometry," Phys. Rev. D 67 (2003) 126005 [arXiv:hep-th/0302127]; K. L. Panigrahi and Sanjay, "D-branes in pp-wave spacetime with nonconstant NS-NS flux," Phys. Lett. B 561 (2003) 284 [arXiv:hep-th/0303182]; N. Ohta, K. L. Panigrahi and Sanjay, "Intersecting branes in pp-wave spacetime," arXiv:hep-th/0306186; R. R. Nayak and K. L. Panigrahi, "More D-branes in plane wave spacetime," arXiv:hep-th/0310219. 
[23] N. D. Lambert and P. C. West, "D-branes in the Green-Schwarz formalism," Phys. Lett. B 459 (1999) 515 [arXiv:hep-th/9905031].

[24] K. Ezawa, Y. Matsuo and K. Murakami, "Matrix regularization of open supermembrane: Towards M-theory five-brane via open supermembrane," Phys. Rev. D 57 (1998) 5118 [arXiv:hep-th/9707200].

[25] B. de Wit, K. Peeters and J. C. Plefka, "Open and closed supermembranes with winding," Nucl. Phys. Proc. Suppl. 68 (1998) 206 [arXiv:hep-th/9710215].

[26] P. Horava and E. Witten, "Heterotic and type I string dynamics from eleven dimensions," Nucl. Phys. B 460 (1996) 506 [arXiv:hep-th/9510209]; "Eleven-Dimensional Supergravity on a Manifold with Boundary," Nucl. Phys. B 475 (1996) 94 [arXiv:hep-th/9603142].

[27] D. Berenstein, J. M. Maldacena and H. Nastase, "Strings in flat space and pp waves from N = 4 super Yang Mills," JHEP 0204 (2002) 013 [arXiv:hep-th/0202021].

[28] B. de Wit, J. Hoppe and H. Nicolai, "On The Quantum Mechanics Of Supermembranes," Nucl. Phys. B 305 (1988) 545.

[29] K. Dasgupta, M. M. Sheikh-Jabbari and M. Van Raamsdonk, "Matrix perturbation theory for M-theory on a PP-wave," JHEP 0205 (2002) 056 [arXiv:hep-th/0205185].

[30] K. Sugiyama and K. Yoshida, "Supermembrane on the pp-wave background," Nucl. Phys. B 644 (2002) 113 [arXiv:hep-th/0206070].

[31] K. Sugiyama and K. Yoshida, "BPS conditions of supermembrane on the pp-wave," Phys. Lett. B 546 (2002) 143 [arXiv:hep-th/0206132];

N. Nakayama, K. Sugiyama and K. Yoshida, "Ground state of the supermembrane on a pp-wave," Phys. Rev. D 68 (2003) 026001 [arXiv:hep-th/0209081].

[32] M. Sakaguchi and K. Yoshida, "Dirichlet branes of the covariant open supermembrane on a pp-wave background," Nucl. Phys. B 676 (2004) 311 [arXiv:hep-th/0306213].

[33] L. Motl, A. Neitzke and M. M. Sheikh-Jabbari, "Heterotic plane wave matrix models and giant gluons," arXiv:hep-th/0306051. 
[34] M. Sakaguchi and K. Yoshida, "Dirichlet Branes of the Covariant Open Supermembrane in $A d S_{4} \times S^{7}$ and $A d S_{7} \times S^{4}$," Nucl. Phys. B 681 (2004) 137 [arXiv:hep-th/0310035].

[35] R. Penrose, "Any spacetime has a plane wave as a limit," Differential geometry and relativity, Reidel, Dordrecht, 1976, pp. 271-275.

R. Gueven, "Plane wave limits and T-duality," Phys. Lett. B 482 (2000) 255 [arXiv:hepth/0005061].

[36] J. Kowalski-Glikman, "Vacuum States In Supersymmetric Kaluza-Klein Theory," Phys. Lett. B 134 (1984) 194.

[37] M. Blau, J. Figueroa-O'Farrill, C. Hull and G. Papadopoulos, "Penrose limits and maximal supersymmetry," Class. Quant. Grav. 19 (2002) L87 [arXiv:hep-th/0201081].

[38] M. Hatsuda, K. Kamimura and M. Sakaguchi, "Nondegenerate super-anti-de Sitter algebra and a superstring action," Phys. Rev. D 62 (2000) 105024 [arXiv:hep-th/0007009]; M. Hatsuda and M. Sakaguchi, "Wess-Zumino term for the AdS superstring and generalized Inoenue-Wigner contraction," Prog. Theor. Phys. 109 (2003) 853 [arXiv:hep-th/0106114]; M. Hatsuda and M. Sakaguchi, "Wess-Zumino term for AdS superstring," Phys. Rev. D 66 (2002) 045020 [arXiv:hep-th/0205092].

[39] M. Hatsuda, K. Kamimura and M. Sakaguchi, "Gauge invariant action for superstring in Ramond-Ramond plane-wave background," Nucl. Phys. B 644 (2002) 40 [arXiv:hepth/0207157].

[40] N. Berkovits and J. Maldacena, " $\mathrm{N}=2$ superconformal description of superstring in Ramond-Ramond plane wave backgrounds," JHEP 0210 (2002) 059 [arXiv:hepth/0208092]; N. Berkovits, "N = 2 sigma models for Ramond-Ramond backgrounds," JHEP 0210 (2002) 071 [arXiv:hep-th/0210078].

[41] M. Cederwall, A. von Gussich, B. E. Nilsson and A. Westerberg, "The Dirichlet superthree-brane in ten-dimensional type IIB supergravity," Nucl. Phys. B 490 (1997) 163 [arXiv:hep-th/9610148]; M. Cederwall, A. von Gussich, B. E. Nilsson, P. Sundell and A. Westerberg, "The Dirichlet super-p-branes in ten-dimensional type IIA and IIB supergravity," Nucl. Phys. B 490 (1997) 179 [arXiv:hep-th/9611159]. 
[42] E. Bergshoeff and P. K. Townsend, "Super D-branes," Nucl. Phys. B 490 (1997) 145 [arXiv:hep-th/9611173].

[43] N. Kim and J. T. Yee, "Supersymmetry and branes in M-theory plane-waves," Phys. Rev. D 67 (2003) 046004 [arXiv:hep-th/0211029].

[44] G. Sarkissian and M. Zamaklar, "Diagonal D-branes in product spaces and their Penrose limits," arXiv:hep-th/0308174.

[45] D. Bak, "Supersymmetric branes in PP wave background," Phys. Rev. D 67 (2003) 045017 [arXiv:hep-th/0204033]; K. Sugiyama and K. Yoshida, "Giant graviton and quantum stability in matrix model on PP-wave background," Phys. Rev. D 66 (2002) 085022 [arXiv:hep-th/0207190]. H. Shin and K. Yoshida, "One-loop flatness of membrane fuzzy sphere interaction in plane-wave matrix model," Nucl. Phys. B 679 (2004) 99 [arXiv:hepth/0309258]; Y. X. Chen and J. Shao, "Giant graviton in type IIA pp-wave background," arXiv:hep-th/0310062; W. H. Huang, "Thermal instability of giant graviton in matrix model on pp-wave background," arXiv:hep-th/0310212; H. Shin and K. Yoshida, "Thermodynamics of fuzzy spheres in pp-wave matrix model," arXiv:hep-th/0401014.

[46] R. R. Metsaev and A. A. Tseytlin, "Supersymmetric D3 brane action in $\mathrm{AdS}_{5} \times S^{5}$," Phys. Lett. B 436 (1998) 281 [arXiv:hep-th/9806095].

[47] N. Itzhaki, I. R. Klebanov and S. Mukhi, "PP wave limit and enhanced supersymmetry in gauge theories," JHEP 0203 (2002) 048 [arXiv:hep-th/0202153]; J. Gomis and H. Ooguri, "Penrose limit of $N=1$ gauge theories," Nucl. Phys. B 635 (2002) 106 [arXiv:hep-th/0202157]; L. A. Pando Zayas and J. Sonnenschein, "On Penrose limits and gauge theories," JHEP 0205 (2002) 010 [arXiv:hep-th/0202186].

[48] M. Sakaguchi and K. Yoshida, in preparation.

[49] D. Berenstein, E. Gava, J. M. Maldacena, K. S. Narain and H. Nastase, "Open strings on plane waves and their Yang-Mills duals," arXiv:hep-th/0203249; V. Balasubramanian, M. x. Huang, T. S. Levi and A. Naqvi, "Open strings from $\mathrm{N}=4$ super Yang-Mills," JHEP 0208 (2002) 037 [arXiv:hep-th/0204196]; P. Lee and J. Park, "Open strings in PP-wave background from defect conformal field theory," Phys. Rev. D 67 (2003) 026002 [arXiv:hep-th/0203257]. 
[50] R. R. Metsaev and A. A. Tseytlin, "Type IIB superstring action in AdS(5) x S(5) background," Nucl. Phys. B 533 (1998) 109 [arXiv:hep-th/9805028].

[51] M. Hatsuda, K. Kamimura and M. Sakaguchi, "From super-AdS $\mathrm{A}_{5} \times S^{5}$ algebra to superpp-wave algebra," Nucl. Phys. B 632 (2002) 114 [arXiv:hep-th/0202190].

[52] M. Hatsuda, K. Kamimura and M. Sakaguchi, "Super-PP-wave algebra from super-AdS $\times$ S algebras in eleven-dimensions," Nucl. Phys. B 637 (2002) 168 [arXiv:hep-th/0204002]. 